\title{
Verfassungsreform als politisches Instrument: Die Einstellung der politischen Eliten Südkoreas zur Verfassung
}

\author{
Von Eun-Jeung Lee*
}

\begin{abstract}
The history of modern constitutions in South Korea began in 1948. According to the 9 th constitutional revision of 1987 currently in force, the Korean people are the constituent power. Nevertheless, in the debate on constitutional reform that set in with democratisation in 1987 the will of the people plays only a minor role. Instead the political elites on purpose stir up debates on constitutional reform as a political gimmick to improve their public standing. One wonders what significance the constitution as such has for Korean politicians. Therefore, in this paper, largely on the basis of reports in the mass media, the topography of the political discourses on constitutional reform during the period 2005-11 is presented. It is shown that traditional and authoritarian traits are still prevalent (of paramount importance) among the political elites. In traditional Korean society the monarch and the political elite established their rule by imposing laws on their subjects. For the South Korean authoritarian rulers before 1987 the constitution was a means to secure and prolong their power. This traditional mode of elitist thinking formed during the authoritarian past is the most fundamental problem and obstacle in the present debate on constitutional reform in South Korea.
\end{abstract}

\section{Problemstellung}

„Verfassungsreform“ ist ein Dauerthema in der südkoreanischen Politik. Seit der Demokratisierung des Landes von 1987 geht diese Diskussion ununterbrochen vonstatten, ohne dass bisher irgendeine Einigkeit darüber besteht, was man wie an der Verfassung ändern will. Die gegensätzlichen Standpunkte erwecken vielmehr den Eindruck, dass sich dadurch die Spaltung zwischen den politischen Lagern vertiefen. Einerseits wird die Notwendigkeit der Änderung der Verfassung betont und dabei behauptet, dass man all seine Kräfte dafür einsetzen müsse, andererseits steht eine gar nicht geringe Zahl von Politikern dieser Diskussion ablehnend gegenüber.

Angesichts dieser Situation fragt man sich, ob die gegenwärtige, 1987 eingeführte Verfassung mit grundsätzlichen Problemen behaftet ist. Bevor man jedoch nach einer juristischtechnischen Antwort sucht, sollte man berücksichtigen, dass der häufigste Einwand gegen

* Professorin für Koreastudien an der Freie Universität Berlin, Eun-Jeung.Lee@fu-berlin.de. Dieser Text wurde für die Konferenz 2011 der Association for Korean Studies in Europe (AKSE) auf Koreanisch verfasst. Mein besonderer Dank gilt Dr. Holmer Brochlos, Institut für Koreastudien, FU Berlin, für die Übersetzung des Manuskripts.

The support of the Academy of Korean Studies, Republic of Korea (AKS-2009-MA-1001) is gratefully acknowledged. 
eine „Verfassungsreform“ lautet, man wolle diese nur aus „,politischem Kalkül“ durchsetzen. Den politischen Eliten wird vorgeworfen, die Debatte um die Verfassungsreform anzuheizen, um sich mit Blick auf die nächsten Präsidentschaftswahlen und die politische Situation danach gewisse Vorteile zu verschaffen. Hier stellt sich die Frage, was südkoreanischen Politikern die Verfassung überhaupt bedeutet. Wodurch ist ihr Verfassungsbild bestimmt? Welchen Stellenwert hat die Verfassungsdiskussion für Politiker und Parteien und wie gehen sie mit ihr um? Welche Rolle spielen die Medien und die koreanische Politikwissenschaft in diesem Zusammenhang?

Die gegenwärtige Verfassung Südkoreas gehört zu den „rigiden“ Verfassungen, die sich als oberstes Gesetz nicht einfach revidieren lassen. Abgesehen davon ist eine Verfassungsänderung an sich eine äußerst sensible politische Angelegenheit. Deshalb kann es unter bestimmten politischen Gegebenheiten und Machtkonstellationen durchaus unangebracht sein, eine Verfassungsdebatte, selbst wenn deren Notwendigkeit außer Zweifel steht, zu initiieren. ${ }^{1}$ Demgegenüber vermittel so mancher Teilnehmer an den Verfassungsdebatten in Korea den Eindruck, als handele es sich hierbei um eine einfache Frage, die sich je nach dem Willen der Politiker entscheiden ließe. Gerade hierin besteht das Kernproblem des sich gegenwärtig in Korea vollziehenden Diskurses über die Verfassungsreform. Man muss sich fragen, ob das aus der Zeit des Autoritarismus stammende Rechtsbewusstsein der politischen Eliten Koreas überwunden worden ist.

In dieser Arbeit werden die Diskurse über die Verfassungsänderung zur Regierungszeit von Roh Moo-hyun (2003-08) und Lee Myung-bak (2008-13) untersucht. Der Grund für diese zeitliche Einschränkung liegt darin, dass Verfassungsänderungen vor dem Machtantritt der Regierung Roh Moo-hyun lediglich von einigen Politikern aus wahltaktischen Gründen thematisiert wurden, und zwar ohne Klärung der verfassungstheoretischen Voraussetzungen und den tatsächlichen Willen, etwas zu ändern. Deshalb stellen einige Wissenschaftler die Ernsthaftigkeit der Diskussionen um eine Verfassungsänderung vor dem Machtantritt von Roh Moo-hyun überhaupt in Frage. ${ }^{2}$ Während vor 2005 verfassungstheoretisch betrachtet keine ernsthafte Entfremdung zwischen Verfassungsnormen und -realität zutage trat, hat sich dies seitdem geändert. Diese Arbeit stützt sich auf von Medien übermittelte Aussagen von Politikern zur Frage der Verfassungsreform, die durch Interviews und die Untersuchung anderer Materialien ergänzt werden. Es werden zunächst einige Besonderheiten in den herkömmlichen koreanischen Auffassungen von Recht und Gesetz erläutert, um dann darzustellen, durch welchen Prozess die gegenwärtige Verfassung Südkoreas in ihrer revidierten Fassung von 1987 zustande gekommen ist und wie sie im folgenden Demokratisierungsprozess thematisiert wurde. Anschließend wird die Topographie des Diskurses um die Verfassungsänderung zwi-

1 Kim Pae-won, Hangukhŏnbŏpsawa hyŏnhaenghŏnbŏp kibonkwonjangŭi kaejŏngbanghyang (Die koreanische Verfassungsgeschichte und grundlegende, richtungsweisende Empfehlungen für eine Revision der gegenwärtigen Verfassung), Kongbŏphak yŏngu 10 (2009) 3, p. 66.

2 Kim Il-hwan, Chisikchŏngbosahoee taebihan hŏnbŏpkaejŏngŭi p'iryosŏnggwa panghyange kwanhan sŏsŏlchŏk koch'al (Interpretative Studie zur Notwendigkeit und Richtung einer Verfassungsreform in der Wissens- und IT-Gesellschaft), Segyehŏnbŏpyŏngu 15 (2009) 2, p. 263. 
schen 2005 und 2011 sowie die sich darin widerspiegelnden Ansichten zur Verfassung untersucht. Es wird sich zeigen, dass das aus autoritären Zeiten stammende traditionelle Rechtsbewusstsein der politischen Eliten das grundlegende Problem in der sich gegenwärtig vollziehenden Verfassungsdebatte darstellt.

\section{Traditionelle Auffassung von der Verfassung in Korea}

Zur Erklärung der Verfassung als Grundgesetz, das in der modernen Gesellschaft die Grundlage sämtlicher gesetzlicher Systeme und Normen bildet, hatte Hans Kelsen den Terminus "Grundnorm“ eingeführt und die Verfassung selbst in untrennbarem Zusammenhang mit der Legitimität des politischen Systems betrachtet. ${ }^{3}$ Die Verfassung als oberstes Gesetz auf der Grundlage des Gemeinwillens der Bürger, d. h. einer volonté générale, bestimmt den grundlegenden Charakter eines Staates. Eine solche Einstellung zur Verfassung entspringt den historischen Erfahrungen Westeuropas. Inmitten der Widersprüche zwischen dem König, der nach absoluter Macht strebte, und den Adligen und Bürgern, die seine Macht beschneiden wollten, entwickelten sich auf der Basis der historischen Erfahrungen Englands, Frankreichs, Deutschlands und anderer europäischer Staaten die nicht-kodifizierte Verfassung Englands und die kontinentalen Verfassungen, die um den Preis des Entrichtens von Steuern die königliche Autorität einschränkten und Grundnormen des Staates festschrieben. In einer solchen Tradition hatte die Verfassung ihren Platz als Grundnorm, die der mit königlicher Oberhoheit identifizierten Staatsmacht bestimmte Schranken setzte, eingenommen. ${ }^{4}$ Auf der Grundlage dieser historischen Erfahrungen entwickelte sich der Begriff der Souveränität des Volkes, der das Grundprinzip der Verfassung eines demokratischen Staates bildet. ${ }^{5}$ Durch eine solche westeuropäische Verfassungsdiskussion wuchs die Erkenntnis, dass das letztendliche Ziel der Schaffung und Änderung einer Verfassung darin besteht, die Grundrechte des Volkes zu stärken und die Macht des Staates zu begrenzen. ${ }^{6}$ In der Frage, wo die Kompetenzen für Verfassungsänderungen liegen, gibt es Unterschiede je nach den historischen Erfahrungen der einzelnen Staaten. ${ }^{7}$

3 Hans Kelsen, Allgemeine Staatslehre, Berlin 1925.

4 Siehe Ernst-Wolfgang Böckenförde, Staat, Verfassung, Demokratie. Studien zur Verfassungstheorie und zum Verfassungsrecht, Frankfurt a. M. 1991, pp. 30-36.

5 Dieser Begriff wurde von Jean-Jacques Rousseau in seinen „Ideen über den Staat“ entwickelt. In seinem Werk „Vom Gesellschaftsvertrag oder Prinzipien des Staatsrechtes“ "stellt er fest: „Ich behaupte also, dass die Souveränität, die nichts anderes als die Ausübung des Allgemeinwillens ist, nie veräußert werden kann und sich der Souverän, der ein kollektives Wesen ist, nur durch sich selbst darstellen lässt. Die Macht kann wohl übertragen werden, aber nicht der Wille." (CS II).

6 Siehe Ernst-Wolfgang Böckenförde, Recht, Staat, Freiheit, Frankfurt a. M. 1991, pp. 217-218.

7 In Deutschland können Änderungen des Grundgesetzes nur mit Zweidrittelmehrheiten des Bundestages und des Bundesrates erfolgen. Änderungen der Bundesverfassung der Schweizerischen Eidgenossenschaft können vom Volk durch eine Initiative oder durch eine der beiden Parlamentskammern vorgeschlagen werden; dieser Vorschlag muss dann sowohl von den Kantonen als auch in einem Volksentscheid angenommen werden. In den USA ist das Verfahren für Verfassungsänderungen 
Die moderne Verfassung Koreas wurde 1948 eingeführt. Nach der gegenwärtig gültigen Verfassung, der 9. revidierten Fassung von 1987, ist das Volk Souverän der Verfassung. Bei der ersten Verfassung und ihren Revisionen haben sich koreanische Politiker und Verfassungsrechtler sehr von Deutschland leiten lassen. In den Interpretationen der Verfassungsrechtler zu Texten und Bestimmungen der koreanischen Verfassung sind die Verbindungen zur deutschen Verfassungslehre leicht zu finden. ${ }^{8}$ Dabei werden die Verfassungsrealität und das Bewusstsein bzw. die Einstellung zur Verfassung, wie auch die sich gegenwärtig vollziehende Verfassungsdebatte zeigt, direkt von den historischen Traditionen und kulturellen Erfahrungen Koreas beeinflusst.

Analysiert man diesen Diskurs, in dem die Politiker einen gewichtigen Part spielen, so kann man in deren Einstellung gegenüber der Verfassung Elemente entdecken, die den Verdacht nahe legen, dass sich diese Ansichten auf der Grundlage ihrer historischen Erfahrungen, insbesondere aus der traditionellen Gesellschaft und der Zeit der autoritären Herrschaft, herausgebildet haben. In der traditionellen koreanischen Gesellschaft, beispielsweise während der Chosŏn-Zeit, machten die Herrscher durch Gesetzeswerke wie das Kyŏngguktaejŏn [Das Große Gesetzesbuch des Landes] ihren Herrschaftsanspruch geltend und verlangten vom Volk sich dem unterzuordnen. ${ }^{9}$ Das Kyŏngguktaejŏn legte die allgemeinen Regeln des Zusammenlebens in Staat und Gesellschaft, einschließlich der Machtstrukturen, fest und kann somit als ein Grundgesetz des Staates bezeichnet werden. Betrachtet man dessen Entstehungsprozess,

komplizierter. Zum einen können Änderungsvorschläge direkt vom Kongress eingebracht werden, zum anderen kann der Kongress auf Antrag von mindestens zwei Dritteln der Bundesstaaten einen Verfassungskonvent einberufen. In beiden Fällen müssen Änderungen dem Kongress zur Verabschiedung vorgelegt werden. Um als offizieller Verfassungsänderungsantrag gültig zu sein, benötigt ein Vorschlag die Zustimmung von mindestens zwei Dritteln der Stimmen beider Kammern. Anschließend müssen die Änderungen mindestens drei Vierteln der Bundesstaaten durch die Parlamente oder speziell zu diesem Zweck zu wählende Versammlungen ratifiziert werden; erst dann treten sie in Kraft. Der Kongress legt dabei fest, ob spezielle Versammlungen zu wählen sind oder nicht.

8 Kim Hyo-jŏn, Hanguge issǒsŏ togil hŏnbŏp ironŭi ch'ogi suyong (Die anfängliche Aufnahme der deutschen Verfassungstheorie in Korea), Tonga taehakkyo taehakwon nonmunjip 2 (1985), pp. 108-142; Kim Hyo-jŏn, Hangugesŏ togil kongbŏp ironŭi yŏnghyang (Der Einfluss deutscher Theorien öffentlichen Rechts in Korea), Handokpŏphak 14 (2003), pp. 331-379; Young Huh, Begegnung europäischer und ostasiatischer Rechtskultur, Verfassung und Recht in Übersee 10 (1977), p. $117 \mathrm{ff}$.

9 Zu Anfang der Chosŏn-Dynastie (1392-1910) erklärten die konfuzianischen Gelehrten „Gesetze zu Mitteln, mit denen der Herrscher das Reich regiert.“ (T'aejosillok Bd. 29, 15. Regierungsjahr von T'aejong, im Mai des Jahres Musin, 2-64, Denkschrift an den König von Sŏng Sŏk-rin u.a.). Das in der 1. Hälfte der Chosŏn-Zeit auf diesem Gedankengut basierende „Kyŏngguktaejŏn“ war in Struktur und Inhalt nicht wesentlich anders als moderne Verfassungen. Die Machteliten, die den Chosŏn-Staat gegründet hatten, wollten durch ein einheitliches Gesetzeswerk einheitliche Normen zur Machtausübung des Staates aufstellen, durch die die Autorität des Staates gestärkt und die Rechtskraft staatlicher Entscheidungen in einer konkreten Form garantiert würden. (To Hyŏn-ch'ǒl, Chogŏnŭi kŏngukkwa yugyomunhwaŭi hwaktae (Reichsgründung unter Konditionen und die Ausweitung der konfuzianischen Kultur), in: O Yŏng-gyo (ed.), Chosŏn kŏngukkwa kyŏngguktaejŏnch'ejeŭi hyŏngsŏng, Seoul 2002, p. 26; Chŏng Ho-hun, Chosŏnjŏngi pŏpchŏnŭi chŏngbiwa kyŏngguktaejŏnŭi sŏngnip (Die Ordnung der Gesetzbücher in der 1. Hälfte der Chosŏn-Dynastie und die Abfassung des Kyŏngguktaejŏn), in: O Yŏng-gyo (ed.), Chosŏn kŏngukkwa kyŏngguktaejŏnch'ejeŭi hyŏngsŏng, Seoul 2002, p. 61. 
wird klar, dass es nicht darum ging, die Macht der Herrscher zu beschränken. Im Gegenteil bringen die politischen Eliten darin vielmehr ihre Ideen über die Verwaltung eines Staates zum Ausdruck. Auch die Diktaturen von Syngman Rhee über Park Chung-hee bis Chun Doohwan benutzten die Verfassung als Mittel zur Sicherung und Verlängerung ihrer politischen Macht. Zu diesem Zwecke wurden von ihnen Verfassungsänderungen nach Belieben vorgenommen. Die in gegenwärtigen Verfassungsdiskursen immer wieder thematisierte Frage nach dahinter liegenden politischen Kalkülen steht in engem Zusammenhang mit diesen historischen Erfahrungen. Unter diesem Gesichtspunkt haben wir die Frage zu stellen, was 60 Jahre nach der Einführung einer modernen Verfassung diese für die politische Elite Koreas bedeutet.

\section{Die Verfassungsgeschichte des modernen Korea: vor und nach 1987}

Die gegenwärtige Verfassung Südkoreas ist das Ergebnis der Demokratisierungsbewegung vom Juni 1987. Sie ist bis heute - darin ist sich die Mehrzahl der Wissenschaftler einig - die tragfähigste Verfassung Koreas. ${ }^{10}$ Die koreanische Verfassung ist seit ihrer ersten Verabschiedung am 17.7.1948 bis 1987 neun Mal revidiert worden. Durchschnittlich blieben sie 4,3 Jahre in Kraft. Die häufigen Revisionen waren nicht Ergebnis von Debatten über sich verändernde Verfassungsrealitäten oder verfassungsrechtlicher Werte. ${ }^{11}$ Um das zu verdeutlichen, soll zunächst ein kurzer Blick auf die Verfassungsgeschichte Südkoreas geworfen werden.

\section{Vor 1987}

Nach dem Ende des Zweiten Weltkrieges und der Befreiung von der japanischen Herrschaft wurde die Koreanische Halbinsel geteilt, und südlich des 38. Breitengrades Südkorea gegründet. Die am 17.7.1948 verabschiedete Verfassung war in einer eigens dafür ins Leben gerufenen Kommission erarbeitet worden und fußte auf dem Präsidialsystem, welches der Vorsitzende der verfassungsgebenden Versammlung, Syngman Rhee, aufgrund der eigenen Ambitionen, Präsident zu werden, bevorzugt hatte. ${ }^{12}$ Gemäß dieser Verfassung sollte der Präsident durch indirekte Wahlen vom Parlament gewählt werden. Syngman Rhee, der dann auch als erster Präsident Südkoreas auf diese Weise gewählt wurde, reichte am 30.11.1951, also mitten im Koreakrieg, beim Parlament einen Antrag zur Verfassungsreform ein, welcher beinhaltete, anstelle eines Einkammer- ein Zweikammersystem einzuführen und den Präsi-

10 Kim Chong-se, Kibon'gwon ilbanjohangŭi paramjikhan hŏnbŏpkaejŏngbanghyang (Wünschenswerte Richtung einer Verfassungsreform der allgemeinen Artikel des Grundgesetzes), Pŏphakyŏngu 34 (2009), p. 19.

11 Sŏ Hyŏn-jin, Minjuhwa ihu hŏnbŏp kaejŏng nonŭiwa kibon'gwon kaejŏng isyu (Die Verfassungsreformdebatte nach der Demokratisierung und die Frage der Revision der Grundrechte), Hyŏndaejŏngch'iyŏngu 3 (2010) 1, p. 132.

12 Kim Su-yong, Kŏngukkwa hŏnbŏp - hŏnbŏpnonŭirŭl t'onghae pon taehanmingukkŏnguksa (Staatsgründung und Verfassung - die Geschichte der Gründung des südkoreanischen Staates durch die Verfassungsdiskussion betrachtet), Seoul 2008, pp. 269-332. 
denten direkt vom Volke wählen zu lassen. Dieser Antrag wurde jedoch abgelehnt. ${ }^{13}$ Der Grund, die noch nicht einmal drei Jahre alte Verfassung zu revidieren, lag darin, dass der Beliebtheitsgrad von Präsident Syngman Rhee gesunken und damit die Wiederwahl durch das Parlament gefährdet war. Nach der Ablehnung dieses Antrags brachte die Opposition, die über die Mehrheit im Parlament verfügte, am 17.4.1952 einen Verfassungsänderungsentwurf für ein Kabinettsystem ein. Als Reaktion darauf legte Syngman Rhee seinerseits am 14.5.1952 einen Revisionsentwurf vor, der ein Direktwahlsystem beinhaltete. Er ließ die Abgeordneten verhaften und in dem während des Krieges in Pusan eingerichteten provisorischen Parlamentsgebäude einsperren, um sodann, mit dem Ziel das Parlament aufzulösen, den Ausnahmezustand zu verhängen. Aufgrund internationaler Proteste ließ es Syngman Rhee dann doch nicht so weit kommen, zumal der Koreakrieg noch nicht beendet war. Er brachte den sogenannten ,selektiven Änderungsantrag“ ein, der den von der Regierung vorgelegten Direktwahlantrag mit dem Vorschlag der Opposition, dessen Misstrauensvotumsklausel gegenüber Kabinettsmitgliedern allerdings gestrichen worden war, verband. Im Zustand der Belagerung des Parlaments durch Militär und Polizei wurde dieser Antrag in einer Abstimmung durch Erheben von den Sitzen verabschiedet. Dieser Vorfall, der als „Politskandal von Pusan“ in die koreanische Verfassungsgeschichte einging, gilt als Paradebeispiel für illegale Verfassungsreformen. ${ }^{14}$

Das Streben Syngman Rhees, am Präsidentenamt festzuhalten, trat mit aller Deutlichkeit im September 1954 zutage, als er in einem neuerlichen Verfassungsreformantrag die Begrenzung auf eine dreimalige Amtszeit des ersten Präsidenten aufheben wollte. Dieser Antrag scheiterte am 17.11., da mit 130 Ja-Stimmen, 60 Gegenstimmen und 7 Enthaltungen eine Stimme zur Zweidrittelmehrheit fehlte. Aber nur zwei Tage später, am 19.11., wurde die Ablehnung für nichtig und der Antrag als angenommen erklärt, und zwar mit der Begründung, dass 2/3 von 203 registrierten Abgeordneten 135,333 Stimmen entsprächen. Da 0,3 Personen keine natürliche Zahl seien, ergäben 2/3 von 203 Abgeordneten durch Rundung 135 Stimmen. Diese als „mathematisch gerundete Revision“ in die Geschichte eingegangene Verfassungsänderung wurde durch die Aprilrevolution von 1960 annulliert.

Syngman Rhee beugte sich schließlich dem Widerstand der Bürger, die sich durch die massiven Wahlfälschungen bei der 4. Präsidentenwahl am 15.3.1960 hintergangen fühlten und zog sich am 24. April vom Amt zurück. Nach seinem Rücktritt brachte die am 2. Mai gebildete Übergangsregierung am 11.6.1960 einen Verfassungsänderungsantrag ein, der ein parlamentarisches Kabinettsystem zum Hauptinhalt hatte. Bei dessen Verabschiedung in der Nationalversammlung am 15. Juni erlebte Korea zum ersten Mal ein verfassungskonformes und demokratisches Verfassungsänderungsverfahren. In Anbetracht der Forderungen nach einer Verurteilung der Rädelsführer der Unrechtswahlen vom 15. März und der Verantwort-

13 Von den anwesenden 163 Abgeordneten stimmten bei 1 Stimmenthaltung 19 mit Ja und 143 mit Nein.

14 Siehe Kim Ch'ŏl-su, Hangukhŏnjŏngsa (Koreanische Verfassungsgeschichte), Seoul 1989; Song U, Hangukhŏnbŏpkaejŏngsa (Geschichte der Verfassungsreform in Korea), Seoul 1980. 
lichen für das in diesem Zusammenhang entstandene Blutvergießen wurde ein weiterer Antrag eingebracht, mit dessen Verabschiedung am 29. November die 4. Verfassungsänderung vollzogen wurde. Sie erlaubte die rückwirkende Bestrafung dieser antidemokratischen Handlungen.

Doch die in einem legalen, demokratischen Verfahren revidierte 4. Verfassung wurde noch nicht einmal ein halbes Jahr nach ihrem Inkrafttreten infolge des Militärputsches vom 16.5.1961 in ihrer Gültigkeit eingeschränkt. Die neue Militärregierung legte fest, dass die Verfassung nur in dem Rahmen gelten dürfte, in dem sie nicht mit dem von ihr erarbeiteten Notstandsgesetz, das sie somit über die Verfassung stellte, in Widerspruch stand. Am 11.7.1962 wurde ein Beratungsausschuss für die neue Verfassung gegründet und diese am 17. Dezember durch eine Volksabstimmung verabschiedet. Ihren Kern bildete eine Direktwahl des Präsidenten, eine vierjährige Amtszeit und die Möglichkeit einer einmaligen Wiederwahl. Die neue Verfassung trat am 27.12.1962 in Kraft.

Auch Park Chung-hee zeigte wie Syngman Rhee zum Ende seiner zweiten Amtszeit keinerlei Absicht, das Amt aufzugeben. Seine Regierung legte deshalb am 7.8.1969 einen weiteren Verfassungsänderungsantrag, der eine dreimalige Wiederwahl erlauben sollte, vor. Dieser Antrag wurde im Morgengrauen des 14. September unter Ausschluss der Opposition, die ihn ablehnte, lediglich von den in einem Nebengebäude des Parlaments versammelten Abgeordneten der Regierungspartei in namentlicher Abstimmung einstimmig angenommen und am 17. Oktober 1969 in einem Volksentscheid bestätigt. Park Chung-hee konnte damit ein drittes Mal gewählt werden. Drei Jahre später rief er am 17.10.1972 den Notstand aus. Schon am 26. Oktober wurde auf einer Notstandssitzung des Kabinetts die „Restaurationsverfassung“ (Yushin-Verfassung) beschlossen. Sie wurde durch eine Volksabstimmung am 21. November bestätigt und am 27. Dezember verkündet. Sämtliche verfassungsmäßigen Instrumentarien, die eine langfristige Herrschaft Park Chung-hees hätten einschränken können, wurden beseitigt.

Die nach der Ermordung Park Chung-hees am 26.10.1979 neuerlich auftretenden demokratischen Hoffnungen wurden zwei Monate später durch die Machtergreifung der Neuen Militärs (sin'gunbu) zunichte gemacht. Chun Doo-hwan, der den erbitterten Widerstand der Bürger Kwangjus im Mai 1980 mit brutaler militärischer Gewalt niedergeschlagen hatte, ließ sich am 1.9.1980 gemäß der Yushin-Verfassung von der T'ongil chuch 'e kungmin hoeŭi (National Conference for Unification) zum 11. Präsidenten wählen. Unter seiner Führung bestätigte das Sonderkomitee für nationale Sicherheitsmaßnahmen (kukka powi pisandaech'aek wiwŏhhoe) am 9. September einen Verfassungsänderungsantrag, der eine einmalige siebenjährige Amtszeit des indirekt gewählten Präsidenten festschrieb. Er wurde am 22. Oktober per Volksentscheid angenommen. Die Verfassung der 5. Republik trat am 27. Oktober in Kraft.

Diesmal regte sich seitens der Bürger Widerstand. Er fand seinen Ausdruck in der Demokratisierungsbewegung. Diese trat 1985 mit der Forderung nach einer Verfassungsänderung zur Direktwahl des Präsidenten zuerst in Erscheinung und kulminierte am 10.6.1987 in großen Massendemonstrationen. Präsident Roh Tae-woo reagierte darauf mit einer 8-Punkte- 
Erklärung vom 29. Juni, in der er in Abstimmung mit der Opposition und im Rahmen einer Verfassungsänderung die friedliche Machtübergabe, die Direktwahl des Präsidenten, eine Generalamnestie und Rehabilitierung aller politischen Gefangenen, Verbesserungen der Medienfreiheit und die Autonomie der Hochschulen versprach. Der daraufhin von Regierung und Opposition erarbeitete Verfassungsänderungsentwurf wurde dem Parlament am 18.9.1987 vorgelegt. Diese, bis heute gültige Verfassung wurde am 27. Oktober in einem Referendum bestätigt und am 29.10.1987 verkündet.

\section{Geschichte der Verfassungsreform}

\begin{tabular}{|l|l|l|}
\hline & Datum & Politischer Anlass \\
\hline Erste Verfassung & 17.7 .1948 & Bildung der Regierung 1948 \\
\hline 1. Verfassungsreform & 7.7 .1952 & $\begin{array}{l}\text { Selektive Verfassungsänderung - Kompromiss zwischen dem Re- } \\
\text { gierungsvorschlag eines Präsidialsystems mit Direktwahl und dem } \\
\text { Vorschlag beider Parteien für ein parlamentarisches Kabinettsystem } \\
\text { - Politskandal von Pusan }\end{array}$ \\
\hline 2. Verfassungsreform & 29.11 .1954 & $\begin{array}{l}\text { „Verfassungsänderung durch Aufrundung“ - erstmalige Einführung } \\
\text { einer dreimaligen Legislaturperiode des Präsidenten }\end{array}$ \\
\hline 3. Verfassungsreform & 15.6 .1960 & $\begin{array}{l}\text { „Revolution vom 19. April“, Einführung des parlamentarischen Sys- } \\
\text { tems }\end{array}$ \\
\hline 4. Verfassungsreform & 29.11 .1960 & $\begin{array}{l}\text { Ergänzung zur Ermöglichung der rückwirkenden Verurteilung anti- } \\
\text { demokratischer Täter }\end{array}$ \\
\hline 5. Verfassungsreform & 26.12 .1962 & Militärputsch vom 16. Mai \\
\hline 6. Verfassungsreform & 21.10 .1969 & Einführung der dreimaligen Amtszeit des Präsidenten \\
\hline 7. Verfassungsreform & 27.12 .1972 & $\begin{array}{l}\text { Interner Militärputsch zur langfristigen Machtabsicherung Park } \\
\text { Chung-hees - die „Erneuerung“ vom Oktober }\end{array}$ \\
\hline 8. Verfassungsreform & 27.10 .1980 & $\begin{array}{l}\text { Militärputsch der „Neuen Militärs“ um Chun Doo-hwan vom } \\
\text { 17. Mai }\end{array}$ \\
\hline 9. Verfassungsreform & 29.10 .1987 & $\begin{array}{l}\text { Demokratischer Widerstand vom 10. Juni und Erklärung vom } \\
\text { 29. Juni }\end{array}$ \\
\hline
\end{tabular}

\section{Die Bedeutung der Verfassung von 1987 und die Folgen}

Wie gezeigt erfolgte die 1. Revision des Grundgesetzes während des kriegsbedingten Ausnahmezustands, ohne jegliche Willensbildung seitens der Bürger, allein aufgrund der Machtgier des Präsidenten. Auch die 2. Revision war eine aufgezwungene, dem Willen der Machthaber folgende, ohne jegliche Übereinkunft mit den Bürgern. Die 3. und die 5. Revision waren Ergebnis der „Revolution vom 19. April“ und des Militärputsches vom 16. Mai. Auch die 7. und die 8. Revision, also die sogenannte „Yushin-Verfassung“ und die Verfassung der 5. Republik, kamen in einer Ausnahmesituation ohne jegliche öffentliche Meinungsbildung zustande. Vor diesem Hintergrund wird allein die Verfassung von 1987 den Standards legitimer Verfassungsänderungen gerecht. 
Das Problem der bis 1987 existierenden Verfassungen ist nicht so sehr in den Verfassungsnormen selbst als vielmehr darin zu suchen, dass die Staatsmacht, die diese Normen umsetzen und befolgen sollte, nicht kontrolliert wurde, zumal in den meisten Fällen die Regierungsbildung ohne ausreichende demokratische Legitimation erfolgt war. ${ }^{15}$ Erst die 1987er Verfassung ermöglichte die friedliche Machtübergabe und den horizontalen Machtwechsel zwischen Regierung und Opposition.

Die Entwicklung der koreanischen Verfassung nach 1987 widerspiegelt den Prozess der Stabilisierung der koreanischen Demokratie. Die Staatsmacht, deren demokratische Legitimation sich auf dem Boden der neuen Verfassung gefestigt hatte, wurde ihrerseits von den Verfassungsnormen kontrolliert, sodass die Verfassung mit Leben erfüllt wurde und die normalen Bürger sie allmählich als normatives Prinzip, das ihre Grundrechte gewährleistet und ihre Rechte schützt, akzeptierten. ${ }^{16}$ Im Zusammenhang damit prägte sich auch das auf der Volkssouveränität fußende Prinzip des Konstitutionalismus, das die Bürger als Quelle der Macht des Staates sieht, tief in das Rechtsbewusstsein der Bürger ein. ${ }^{17}$

In der koreanischen Gesellschaft, in der der Terminus „Verfassungspatriotismus“ ungewohnt klingt, stimmen die meisten Wissenschaftler und Politiker wenigstens in dem Punkt überein, dass man stolz darauf sein kann, dass die Verfassung von 1987 durch den demokratischen Widerstand der Bürger erkämpft worden ist. Obendrein hat keine andere Verfassung so lange gehalten und so viel zur Verwurzelung der Demokratie in der politischen Realität Koreas beigetragen. Sie gilt deshalb als die bisher beste koreanische Verfassung. ${ }^{18}$

Dennoch wird bisweilen die Tatsache problematisiert, dass die 1987er Verfassung in nur kurzer Zeit und ohne einen breit angelegten Prozess der öffentlichen Meinungsbildung zustande gekommen ist. ${ }^{19}$ Aus verfassungsrechtlicher Sicht sind die Leistungen der neuen Ver-

15 In diesem Zusammenhang sollte die Aussage des Verfassungsrechtlers Konrad Hesse (Die normative Kraft der Verfassung. Freiburger Antrittsvorlesung, Recht und Staat in Geschichte und Gegenwart, Tübingen 1959, p. 4) beachtet werden, dass „,die Verfassungsgeschichte der einzelnen Staaten anschaulich zeigt, dass die Kraft der politischen Realität stets stärker war als die der gesetzlichen Normen oder anders gesagt, dass sich der normative Charakter stets den Realitäten unterordnen musste.“ In diesem Sinne bildet die Verfassungsgeschichte Koreas keine Ausnahme.

16 Kim, note 2, p. 257.

17 Ein Beispiel für dieses Rechtsbewusstsein ist das Lied „Artikel 1 der Verfassung“, das während der Mitte 2008 mehr als drei Monate andauernden Kerzendemonstrationen intoniert wurde. Dieser Artikel 1 „Südkorea ist eine demokratische Republik. Alle Macht geht vom Volke aus.“ war mit einer einfachen Melodie unterlegt worden, sodass alle leicht mitsingen konnte.

18 Sŏ Hyŏn-jin, Minjuhwa ihu hŏnbŏp kaejŏng nonŭiwa kibon'gwon kaejŏng isyu (Die Debatte um Verfassungsreform nach der Demokratisierung und die Frage der Revision der Grundrechte), Hyŏndaejŏngch'iyŏngu 3 (2010) 1, p. 133.

19 Chŏng Man-hŭi, Hŏnbŏp kaejŏngnonŭi - hŏnbŏpkaejŏngŭi p'iryosŏnggwa, kaehŏnjŏlch'a pangbŏbe kwanhan kŏmt'o (Die Debatte um Verfassungsreform - ihre Notwendigkeit und die Überprüfung ihrer Verfahrensmethoden), Tongabŏphak 47 (2010), p. 13. Man kann hier die Gegenfrage stellen, in wie vielen Fällen es gelungen ist, Verfassungen ,unter normalen Umständen“ und mit ausreichend Zeit zustande zu bringen. In Frankreich entstand die Verfassung inmitten der Revolution, während sie in den USA in der Begeisterung des Sieges im Unabhängigkeitskrieg geschrieben wurde. In Japan 
fassung zwar anerkennenswert, doch sei es auch zu gravierenden systematischen Verzerrungen gekommen, da die Verfassung durch Vereinbarungen zwischen Politikern entstanden und Experten kaum konsultiert worden seien. ${ }^{20}$ So wird von Politikwissenschaftlern häufig die Meinung vertreten, dass die einmalige Amtszeit von fünf Jahren zu kurz sei und der Verkörperung verantwortungsvoller Politik entgegen stünde. ${ }^{21}$

Unabhängig von solchen akademischen Einlassungen konnte sich die Verfassungsreform seit Anfang der 1990er Jahre als ein wichtiges Thema in der koreanischen Politik etablieren. Das begann damit, dass der Vorsitzende der Demokratischen Gerechtigkeitspartei (Minju chŏngŭidang, DJP), Präsident Roh Tae-woo, der Vorsitzende der Demokratischen Partei, Kim Young-sam, und der Vorsitzende der Neuen Demokratisch-Republikanischen Partei, Kim Jong-pil, beim Zusammenschluss dieser drei Parteien zur Demokratisch-Liberalen Partei am 9. Februar 1990 eine geheime Absprache getroffen hatten, ein Kabinettsystem einzuführen. Jeder dieser drei Politiker hatte aus eigenem politischem Kalkül heraus die Vereinigung der drei Parteien vorangetrieben. Präsident Roh Tae-woo, der an der Niederschlagung des Aufstands von Kwangju 1980 beteiligt gewesen war, versprach sich davon, Schutz nach dem Ende seiner Amtszeit und meinte, die alten Kräfte stärken zu können. Kim Young-sam hingegen wollte mit dem Zusammenschluss der drei Parteien ein günstiges politisches Umfeld für die nächste Präsidentenwahl schaffen, während sich Kim Jong-pil davon eine Stärkung seiner Minderheitspartei erhoffte. Für sie war die Änderung der Verfassung hin zu einem Kabinettsystem sehr attraktiv. Allerdings gelangte ihre geheime Vereinbarung durch Machtkämpfe zwischen ihren Fraktionen innerhalb der neuen Partei am 25. Oktober in die Öffentlichkeit und musste schließlich am 6. November aufgehoben werden.

Auch im Verlaufe der Präsidentschaftswahlen von 1997 wurde die Verfassungsreform in ähnlicher Form thematisiert. Im Vorlauf hatten die beiden Kandidaten Kim Dae-jung und Kim Jong-pil ein Wahlbündnis gebildet und an zwei Bedingungen, nämlich die Aufstellung eines gemeinsamen Kandidaten und einer Verfassungsänderung zur Schaffung eines Kabinettsystems, geknüpft. Diese wurde dieses Mal nicht als geheimes Einvernehmen, sondern als offizielle Politik des Wahlbündnisses definiert. Kim Dae-jung, der dreimal (1970, 1987 und 1992) bei Präsidentenwahlen gescheitert war und sich unmittelbar nach der Wahl 1992 aus der Politik zurückgezogen hatte, erklärte im Juli 1995 seine Rückkehr in die Politik und gründete im September den „Volkskongress für Neue Politik“(Saechŏngch’i kungmin hoeŭi). Die Wahl im Jahre 1997 war für ihn zweifellos die letzte Gelegenheit, Präsident zu werden. Da er einen Wahlsieg nur im Verbund mit der Opposition und der Aufstellung eines einheitlichen Kandidaten für möglich hielt, suchte er ein Bündnis mit Kim Jong-pil einzugehen. Weil für diesen

wurde die Friedensverfassung nach der Kapitulation unter recht eigentümlichen Verhältnissen verfasst und verabschiedet. Historisch betrachtet sind Verfassungen meist unter Bedingungen entstanden, die man ganz sicher nicht als ruhig bezeichnen kann (Hasebe Yasuo / Sugita Atsushi, Hŏnbŏpnonjaeng (Verfassungsdiskussion), Seoul 2006, p. 31).

20 Chŏng, note 19, p. 14

21 Cho Chi-hyŏng, Hŏnbŏbe pich'in yŏksa (Geschichte im Spiegel der Verfassung), Seoul 2007, pp. 26-27. 
als Vorsitzenden einer Minderheitspartei das Kabinettsystem nach wie vor eine attraktive Option darstellte, stimmte auch er dem Bündnis zu. Doch so wie die von Roh Tae-woo, Kim Young-sam und Kim Jong-pil vereinbarte Schaffung eines Kabinettsystems nach dem Parteizusammenschluss wieder annulliert worden war, wurde auch diese Vereinbarung zwischen Kim Dae-jung und Kim Jong-pil nicht eingehalten. Kim Dae-jung lehnte nach seinem Amtsantritt im Februar 1998 ebenso wie die Demokratische Partei eine Verfassungsänderung ab, da man wegen der Asienkrise alle Hände voll zu tun hätte und die Oppositionspartei, die Grand National Party (Hannaradang, GNP), ohne die keine ausreichende Mehrheit möglich war, dagegen wäre. Als sich im September 2001 bei der Abstimmung über die Entlassung des Ministers für Wiedervereinigung, Lim Dong-won, die United Liberal Democrats (Chayuminju yŏnhap ULD) auf die Seite der GNP schlug, kam es zudem zum Bruch mit der Demokratischen Partei. An eine Verfassungsänderung war nun gar nicht mehr zu denken.

Während der Präsidentschaftswahlkampfes 2002 bildete die Verfassungsreform erneut eines der Hauptthemen. Roh Moo-hyun, der Präsidentschaftskandidat der Demokratischen Partei, und der Vorsitzende der „Partei der Nationalen Einheit 21“ (Kungmin t'onghap 21), Chung Mong-joon, vereinbarten unter der Voraussetzung eines Präsidialsystems, in dem die Macht des Präsidenten eingeschränkt und die Position des Ministerpräsidenten gestärkt wird, und einer kurzfristig anzuberaumenden Verfassungsreform ihre gegenseitige Unterstützung bei den Wahlen und veröffentlichten am 12.12.2002 eine gemeinsame Erklärung zur Zusammenarbeit. Doch am 18. Dezember, unmittelbar vor den Wahlen, zog Chung Mong-joon seine Unterstützung der Kandidatur Roh Moo-hyuns zurück und ließ damit auch die Verfassungsreform hinfällig werden.

Roh Moo-hyun verfolgte das Vorhaben trotzdem weiter und äußerte unmittelbar nach seiner Wahl zum Präsidenten, dass es wünschenswert wäre, noch 2006 mit der Diskussion um die Verfassungsänderung zu beginnen. Man habe zwar bei der Entscheidung für ein reines Präsidialsystem oder ein dezentralisiertes Kabinettsystem dem Willen des Volkes zu folgen, doch müsste die Verfassungsreform bis Ende 2006 abgeschlossen werden, damit die Wahlen 2007 auf dieser Basis durchgeführt werden könnten. “22 Noch vor den allgemeinen Wahlen zur Nationalversammlung 2004 sollte ein Kabinettsystem oder ein dezentralisiertes Präsidialsystem eingeführt werden. Die Oppositionsparteien GNP und ULD favorisierten im Einklang mit einer Abgeordnetengruppe, die Roh Moo-hyun unterstützte und sich in der „Neuen Partei der Nationalen Einheit" (Kungmin t'onghap sindang) zusammengeschlossen hatte, ein Kabinettsystem, während die restlichen Abgeordneten seiner Partei ein System mit einem verantwortlichen Premierministers anstrebte. Im Vorfeld der Parlamentswahlen vom 15.4.2004 lobte Präsident Roh Moo-hyun das gemeinsame Bemühen von Demokratischer Partei, GNP und ULD um eine Verfassungsreform und verband damit seinen Wunsch an die Wähler, die „Neue Partei der Nationalen Einheit“ zu stärken. Aufgrund dieser Äußerung wurden ihm ungesetzliche Wahlkampfmethoden vorgeworfen, die schließlich zur überhaupt ersten Einleitung eines Amtsenthebungsverfahrens in der Nationalversammlung führten. Die 
öffentliche Meinung reagierte darauf allerdings so heftig, dass die neu gegründete „Our Open Party“ (Yóllin uridang OOP), die den Präsidenten unterstützte, bei den Wahlen am 15. April die absolute Mehrheit gewinnen und zur führenden Partei werden konnte. Allerdings kam die Debatte um eine Verfassungsreform dadurch eine Zeitlang zum Stillstand.

2005 schlug Präsident Roh Moo-hyun eine große Koalition mit der Opposition vor und brachte durch seine Äußerung, dass er, sofern die politischen Kräfte einverstanden seien, eine Neuordnung der Wahlbezirke zu akzeptieren, „,bereit sei, die Rechte des Präsidenten auf das Niveau eines Kabinettsystems zu übertragen“, die Verfassungsdebatte wieder ins Spiel. Er sei sogar bereit, das Präsidentenamt aufzugeben. ${ }^{23}$ Die Oppositionspartei GNP warf ihm allerdings politisches Taktieren vor, um bis zum Ende der Legislaturperiode eine Führungsrolle in Staatsangelegenheiten behalten zu können. Am 7.9.2005 wies die Vorsitzende der GNP, Park Geun-hye, ${ }^{24}$ diesen Vorschlag offiziell zurück und beendete damit die Verfassungsdebatte.

In einer Erklärung am 9.1.2007 gab Präsident Roh Moo-hyun bekannt, dass er einen Antrag auf Verfassungsänderung einreichen werde, der nur aus einem Punkt bestehen würde, und zwar der Reduzierung der Amtszeit des Präsidenten auf vier Jahre in Verbindung mit der Möglichkeit einer einmaligen Amtsverlängerung. Am 8.3.2007 veröffentlichte die mit der Verfassungsreform beauftragte Regierungskommission einen Vorschlag, der im Kern die Einführung einer vierjährigen, einmalig verlängerbaren Amtszeit des Präsidenten sowie die zeitgleiche Durchführung der Präsidentenwahl und der Parlamentswahlen beinhaltete und die Vorstellungen Präsident Rohs konkrete Gestalt annehmen ließ. Dieser Versuch der Regierung stieß allerdings auf Ablehnung bei der Opposition, die politische Absichten des Präsidenten vermutete. Am 11. April verständigten sich die Abgeordneten von sechs politischen Gruppierungen, also den fünf Parteien (GNP, OOP, Demokratische Partei, Demokratische Arbeiterpartei und People First Party) und der „Vereinigung zur Gründung einer neuen Einheitspartei“, darauf, Präsident Roh zu bitten, während seiner Amtszeit auf weitere Vorschläge zur Verfassungsänderung zu verzichten. Im Gegenzug vereinbarten sie, diese Frage zu Beginn der 18. Legislaturperiode der Nationalversammlung zu behandeln. Präsident Roh Moo-hyun erwiderte darauf, er werde seinen Vorschlag zur Verfassungsreform zurückziehen, sofern jede Partei sich programmatisch dazu verpflichtete, zu Beginn der 18. Nationalversammlung eine Verfassungsreform zur Verwirklichung einer vierjährigen verlängerbaren Amtszeit des Präsidenten einzubringen. Am 13.4.2007 wurde dies von den GNP-Abgeordneten formal akzeptiert. Am folgenden Tag erklärte der Präsident, dass er die Vereinbarung der politischen Parteien annehme. Damit war die Diskussion um eine Verfassungsänderung wieder einmal beendet. Es war absehbar, dass ihre Verpflichtung für eine Fortsetzung der Debatte sorgen würde.

23 Gespräch mit dem Chef der Nachrichtenabteilung der Redaktion des Chungang Medienverlags am 7.7.2005; Journalistengespräch am 29.7.2005 (Yonhap News Agency, 28.11.2006).

24 Sie ist am 19.12.2012 zur Präsidentin gewählt worden (2013-18). 
Auf der Gedenkveranstaltung zum Verfassungstag am 17.7.2009 schlug Parlamentspräsident Kim Hyŏng-o die Gründung eines Sonderausschusses zur Verfassungsreform unter Beteiligung von Regierung und Opposition vor. Wenig später wurde das Parlament von Präsident Lee Myung-bak auf der Gedenkveranstaltung zum Tag der Befreiung am 15.8.2009 aufgefordert, sich mit dieser Verfassungsreform zu befassen. Im Juli 2008 war zu diesem Zweck bereits die „Arbeitsgemeinschaft für eine zukünftige Verfassung Koreas“ (Mirae Han'guk hŏnbŏp yŏn 'guhoe $)^{25}$ mit Abgeordneten von Regierung und Opposition gegründet worden. Darüber hinaus wurde 2009 vom Beratenden Ausschuss für Verfassungsstudien (Hŏnbŏp yŏn'gu chamun wiwŏnhoe) des Parlaments, der sich vor allem aus Verfassungsrechtlern und Politikwissenschaftlern zusammensetzte, ein Bericht vorgelegt.

Die mit dem Amtsantritt von Lee Myung-bak zur Regierungspartei avancierte GNP favorisiert ein dezentrales Präsidialsystem, in dem die Rechte des Präsidenten beschränkt sind und der Premier die Hauptverantwortung trägt. Die Diskussion wird in einer bisher nie dagewesenen Breite nicht nur in akademischen, sondern auch zivilgesellschaftlichen Kreisen geführt. Zu Notwendigkeit und Inhalt sowie zur Grundrichtung der Verfassungsänderung werden die unterschiedlichsten Meinungen vorgetragen. Dadurch steht den Bürgern eine gute Grundlage zu ihrer Meinungsbildung zur Verfügung. ${ }^{26}$

\section{Anläufe zur Verfassungsreform seit der Demokratisierung}

\begin{tabular}{|l|l|l|}
\hline Zeit & Akteure & Themen \\
\hline $\begin{array}{l}\text { Jan. - } \\
\text { Nov. 1990 }\end{array}$ & $\begin{array}{l}\text { DJP, Dem. Partei, Neue Dem.- } \\
\text { Rep. Partei; Roh Tae-woo, Kim } \\
\text { Young-sam, Kim Jong-pil }\end{array}$ & $\begin{array}{l}\text { Zusammenschluss von drei Par- } \\
\text { teien und Geheimabsprache „Ka- } \\
\text { binettsystem“ }\end{array}$ \\
\hline $\begin{array}{l}\text { Okt. 1997 - } \\
1999\end{array}$ & Kim Dae-jung, Kim Jong-pil & $\begin{array}{l}\text { Wahlbündnis der DJP und Ver- } \\
\text { einbarung „Kabinettsystem“ }\end{array}$ \\
\hline $\begin{array}{l}\text { Nov. - Dez. } \\
2002\end{array}$ & $\begin{array}{l}\text { Roh Moo-hyun, Chung Mong- } \\
\text { joon }\end{array}$ & $\begin{array}{l}\text { Wahlsieg und Verfassungsre- } \\
\text { form im S. eines dezentralen Prä- } \\
\text { sidialsystems }\end{array}$ \\
\hline $\begin{array}{l}\text { Sept. 2003- } \\
\text { April 2004 }\end{array}$ & $\begin{array}{l}\text { Pro-Roh, Anti-Roh (Dem. Ein- } \\
\text { heitspartei, Dem. Partei, GNP, } \\
\text { ULD) }\end{array}$ & $\begin{array}{l}\text { Verfassungsreform „Verant- } \\
\text { wortliches Kabinettsystem“ oder } \\
\text { „Dezentrales Präsidialsystem““ }\end{array}$ \\
\hline $\begin{array}{l}\text { Juni- Sept. } \\
2005\end{array}$ & Präsident Roh Moo-hyun & $\begin{array}{l}\text { Vorschlag Große Koalition und } \\
\text { Verfassungsreform „Kabinett- } \\
\text { system“ }\end{array}$ \\
\hline
\end{tabular}

26 Einige Autoren sehen darin allerdings eher einen Beitrag zur Verwirrung der Bürger (Chŏng, note 19, p. 45). 


\begin{tabular}{|l|l|l|}
\hline Zeit & Akteure & Themen \\
\hline $\begin{array}{l}\text { Jan. - April } \\
2007\end{array}$ & Präsident Roh Moo-hyun & $\begin{array}{l}\text { Ein-Punkt-Vorschlag zur Ver- } \\
\text { fassungsreform: vierjährige, ver- } \\
\text { längerbare Amtszeit }\end{array}$ \\
\hline seit 2009 & $\begin{array}{l}\text { Präsident Lee Myung-bak, Pro- } \\
\text { LMB-Fraktion, GNP }\end{array}$ & $\begin{array}{l}\text { Eingeschränktes Präsidialsystem } \\
\text { mit einem starken Ministerpräsi- } \\
\text { denten }\end{array}$ \\
\hline
\end{tabular}

\section{Topographie des Diskurses zur Verfassungsreform}

Im gegenwärtigen Diskurs zur Verfassungsreform finden sich mannigfaltige Narrative zu Grundrechten, Rechtsprinzipien, Reichweiten und Grenzen von Macht, Machtverhältnissen und Interessenkoalitionen. Auch die Akteure, insbesondere Politiker und Medien, greifen gerne auf diese, nicht unbedingt untereinander konsistenten und auch nur teilweise überlappenden Narrative zurück, um ihre Positionen zu untermauern. Diese Segmentierung hat dazu beigetragen, dass kein stimulierender Diskursverbund entstanden ist und der Diskurs selbst zu keiner Entscheidung oder Abschluss geführt wird. Deshalb flammt er auch immer wieder auf.

Bei den Debatten um die Verfassungsreform ging es nach 1987 im Kern stets um die Neuordnung der Machtverhältnisse im politischen System des Landes. Von der Öffentlichkeit wurden diese Debatten genau wie in der Zeit der autoritären Regime weitgehend als auf parteilicher Rhetorik basierende Überlegungen wahrgenommen. Auch die Akteure selbst werfen sich gerne gegenseitig vor, dass es kein gemeinsames Bemühen zur Ausarbeitung von grundlegenden Normen für die Zukunft des Staates, sondern nur ,„parteipolitisch begründete Auseinandersetzungen“ gebe, um damit ihren eigenen Führungsanspruch zu begründen. In der dahinter liegenden kompromisslosen Entscheidungslogik kann man das Wirken Carl Schmitts, der unter koreanischen Verfassungsrechtlern viele Anhänger hat, ${ }^{27}$ erkennen. In

27 Siehe Kwon Yŏng-sang, Hŏnbŏpkaejŏngŭi pŏmni (Rechtsprinzipien der Verfassungsreform), Sŏultaehakkyo pŏphak 19 (1979) 1, p. 259; Kal Bongkun, Carl Schmitts Einfluss auf das koreanische Verfassungsleben, in: Helmut Quaritsch (ed.), Complexio Oppositorum. Über Carl Schmitt, Berlin 1988, pp. 503-507; Kim Hyo-jin, K'alsyumit'ŭ hŏnbŏp ironŭi hangukchŏk chŏngae (Die Verbreitung von Carl Schmitts Verfassungstheorie in Korea), in: Kim Hyo-jin (ed.) Togilhŏnbŏphaksŏlsa (Geschichte der deutschen Verfassungslehre), Seoul 1982, pp. 125-174; Kim Sang-gi, K'al syumit'ǔwa uriŭi chŏngch'ijŏk hyŏnsil (Carl Schmitt und unsere politische Realität), Ch'ŏrhakkwa hyŏnsil 5 (1990), pp. 238-253; Pae Sang-ho / Mun Chŏng-guk, K'alsyumit’ǔŭi hŏnbŏp kaenyŏmŭi hangukchŏk chŏngae (Die Verbreitung von Carl Schmitts Verfassungsbegriff in Korea), Pŏphakyŏngu 6 (1995) 1, pp. 25-50; Eun-Jeung Lee, Carl Schmitt Rezeption in Korea, in: Rüdiger Voigt (ed.), Internationale Rezeption von Carl Schmitt in der Gegenwart, Baden-Baden 2007, pp. 239-260. 
dieser Logik kommt es weniger auf gemeinschaftlichen Konsens als vielmehr auf ,politische Entscheidungen" und ihre Durchsetzung an. ${ }^{28}$

Dies hat zu der seltsamen Erscheinung geführt, dass die Verfassungsdiskussionen nicht an einem Ort des Meinungsaustausches, sondern an mehreren Diskursorten, an denen Gruppen mit ähnlichen Meinungen jeweils ihre eigenen Geschichten präsentieren, geführt werden. Der koreanische Diskurs zur Verfassungsreform zeigt keine Entwicklung in der Form, dass unterschiedliche Auffassungen miteinander wetteifern und ein Diskursverbund, der zur Konsensbildung beiträgt und die politische Entscheidungsfindung erleichtert, gebildet wird. Deshalb soll im Folgenden kurz auf die Topographie der Diskurse in Politik, Wissenschaft und Zivilgesellschaft eingegangen werden.

Neben Politikern beteiligen sich Verfassungsrechtler, Politologen, zivilgesellschaftliche Verbände sowie Medien aktiv am Verfassungsdiskurs. Die Diskursstruktur der Wissenschaftler und der zivilgesellschaftlichen Gruppen ist vergleichsweise klar. Unter Wissenschaftlern gibt es solche, die die Notwendigkeit der Änderung der gegenwärtigen Verfassung vertreten und andere die keinen dringenden Änderungsbedarf erkennen. Die Befürworter verbreiten ihre Auffassungen über die Medien und Veröffentlichungen. Von den zivilgesellschaftlichen Gruppen, ganz gleich ob dem konservativen oder dem fortschrittlichen Lager zugehörig, beteiligen sich vor allem diejenigen aktiv am Diskurs, die die Notwendigkeit einer Verfassungsänderung in welcher Form auch immer bejahen. ${ }^{29}$

Um die mittlerweile 25 Jahre alte Verfassung reformieren zu wollen er, bedarf es einer überzeugenden Argumentation. 25 Jahre mögen verglichen mit anderen Ländern ein kurzer Zeitraum sein, doch geben sie dieser Verfassung von 1987 angesichts der häufigen Revisionen in der Zeit davor eine gewisse Alterswürde. Dennoch finden sich Befürworter einer Reform, die schlicht davon ausgehen, dass eine über 20 Jahre alte Verfassung nicht mehr mit der Realität übereinstimmen kann. So stellt das „Beratende Gremium für Verfassungsfragen“ des Parlaments in einem Bericht fest, dass ,die gegenwärtige Verfassung zwar, erfüllt von der Sehnsucht der Bürger nach Demokratie, zur Grundlage der tatsächlichen Erweiterung ihrer Grundrechte und der Auflösung des autoritären Systems geworden ist, in den vergangenen

28 Hŏ Hwa-p'yŏng, ein politischer Wegbegleiter Chun Doo-hwans, begründete dies mit der Notwendigkeit der Überwindung der politischen Verhältnisse, die sich seit den 1990er Jahren entwickelt haben. Dafür sei es erforderlich, zunächst ,die ideologischen Werte festzulegen, die in der Verfassung zu nennen sind, die beiden Seiten klar zu unterscheiden und die falschen, unnötigen bzw. vermeidbaren Ansichten über Diskussionen und Wortstreitereien über Bord zu werfen." Hier zeigt sich, wie verhärtet das politische Denken koreanischer Konservative sein kann und wie sehr es Schmittschem Denken durchdrungen ist. Siehe Hŏ Hwa-p'yŏng, Kajang kŭnwonjŏgin kŏse taehayŏ - Hŏ Hwap'yŏngŭi kaehŏnch'ŏngwonron (Über die grundlegendsten Dinge - Hŏ Hwa-p'yŏng's theoretischer Diskurs über den Wunsch nach Verfassungsreform), Seoul 2011, p. 223.

29 Ein Beispiel sind die von der Daehwa Munhwa Akademie von April 2006 bis April 2008 veranstalteten Diskussionsforen. Die sich dort versammelnden einflussreichen Intellektuelle zeigten sich bei aller Wertschätzung für die Verfassung von 1987 einig, dass diese etwa den inzwischen ganz veränderten gesellschaftlichen Verhältnissen nicht mehr gerecht werden können. Die Ergebnisse dieser Diskussionen wurden 2008 unter dem Titel „Saeroun hŏnbŏp p'iryohanga“ (Brauchen wir eine neue Verfassung?) veröffentlicht. 
mehr als 20 Jahren aber infolge drastischer Veränderungen des politischen, ökonomischen und sozialen Umfelds im In- und Ausland allerorts Probleme zutage getreten sind, die sich mit herkömmlichen Denkvorstellungen und Paradigmen nicht lösen lassen, sodass sich eine das gesamte staatliche System erfassende erneute Überprüfung in verfassungsmäßiger Hinsicht als unumgänglich erwiesen hat." Insbesondere in Bezug auf die Machtstrukturen, dem Kernbereich der Verfassungsreform, wird diese als notwendig erachtet, „um Problempunkte zu korrigieren, die sich ununterbrochen ergeben haben, wie beispielsweise durch Machtkonzentration verursachte negative Konsequenzen beim verfassungsmäßigen Ausüben der Regierungsgewalt, durch die fünfjährige einmalige Amtszeit bedingte Schwächung der politischen Verantwortung sowie Behinderungen der nationalen Einheit infolge übertriebenen Machtwettstreits, und um mittels wohldurchdachter Teilung der Macht und anderer Maßnahmen das Rückgrat des staatlichen Systems zu reformieren.“30

Im Gegensatz dazu gibt es auch den Fall, dass man sich am Diskurs zur Verfassungsreform beteiligt, sie aber nur eingeschränkt befürwortet. So erklärt der Politikwissenschaftler Pak Ch'an-uk: „Ich kann mich dem Standpunkt einer Verfassungsreform hin zu einem dezentralen Präsidialsystem oder einem Kabinettsystem nicht anschließen. Selbstverständlich erkenne ich vollkommen an, dass es Unzulänglichkeiten hinsichtlich der Führungsqualitäten von Präsident Roh Moo-hyun gibt, und dass die Erfolge seiner Regierung noch nicht klar erkennbar sind. Auch stimme ich zu, dass es nicht wenige unvollkommene Punkte in den Bestimmungen der gegenwärtigen Verfassung bezüglich der Machtstruktur gibt. Trotzdem denke ich nicht einfach, dass diese Bestimmungen das grundlegende Übel, die Ursache Nummer Eins für die konfuse Situation der Verhältnisse im Lande sind. Da noch andere politisch-institutionelle und situationsbezogene Bedingungen, unter denen zumindest die derzeitigen Bestimmungen der Machtstruktur ordnungsgemäß funktionieren könnten, unzulänglich sind, sollte nicht übersehen werden, dass diese verbessert werden müssen. ... Falls zusammen mit der oben dargelegten Meinung die Diskussion zur Verfassungsreform konkretisiert wird, ... bin ich richtungsmäßig betrachtet ganz entschieden gegen ein dezentralisiertes Präsidialsystem und eher der Auffassung, dass es wünschenswerter ist, näher am reinen Präsidialsystem zu bleiben als ein Kabinettsystem zu favorisieren." ${ }^{\text {(31 }}$

\section{Wissenschaft und Zivilgesellschaft}

Die Argumente der Politikwissenschaftler, die sich aktiv an der Verfassungsdiskussion beteiligen, sind ähnlich wie bei Politikern auf die Machtstrukturen fokussiert. Es geht hauptsächlich um Dinge wie Pro und Kontra eines Übergangs zum Kabinettsystem bzw. bei Beibehaltung des Präsidialsystems um Beschränkungen der Amtszeit, die Möglichkeit der Wie-

30 Beratergremium des Parlamentspräsidenten: Hŏnbŏpyŏngujamunwiwonhoe kyŏlkwabogosŏ (Bericht des Beratenden Ausschusses für Verfassungsstudien), August 2008, S. 3.

31 Pak Ch'an-uk, Taet'ongryŏngjeŭi chŏngsangjŏk chaktongŭl wihan kaehŏnron (Verfassungsreform für ein funktionierendes Präsidialsystem), in: Chin Yŏng-jae (ed.), Hanguk kwollyŏkkujoŭi ihae (Verständnis der Machtstrukturen in Südkorea), Seoul 2004, p. 179. 
derwahl, die Abschaffung des Postens des Ministerpräsidenten sowie die Einführung eines Vizepräsidentenamtes. ${ }^{32}$ Die Debatten unter Verfassungsrechtlern sind naturgemäß breiter angelegt. Sie befassen sich zusätzlich mit Struktur und System der Organisation des Verfassungstextes, Grundrechten, Fragen der Wirtschaft und der lokalen Selbstverwaltung und umfassen somit sämtliche Bereiche der Verfassung. ${ }^{33}$

Auch von fortschrittlichen zivilgesellschaftlichen Verbänden wird, unter Anerkennung der gegenwärtigen Verfassung als Erfolg des demokratischen Widerstands, die Meinung vertreten, dass eine umfassende Verfassungsreform anzustreben sei. Eine Kernfrage, wie sie am 15.7.2005 auf dem gemeinsam vom Verlag „Ch'angjakkwa pip’yŏng“ und der „Bürgeraktion", einer Versammlung progressiver zivilgesellschaftlicher Verbände, veranstalteten Symposium „Für die Überwindung des Systems von 1987 - kritische Betrachtung von Verfassung und Sozialstruktur" diskutiert wurde, bildete die genauere Bestimmung der sozialen und politischen Positionen der gegenwärtigen Verfassung und der in ihr verborgenen Elemente klassenmäßiger Diskriminierung. ${ }^{34}$

32 Z.B. Pak, note 30, pp. 171-223; Kim Yong-ho, 2003nyŏn hŏnjŏngwigiŭi woningwa ch'ŏbang. Jesamdang punjŏmjŏngbuwa taet'ongrŏng-kukhoe kanŭi taerip (Ursachen und Lösungsmöglichkeiten der Krise der verfassungsmäßigen Regierung im Jahre 2003. Kohabitation und der Konflikt zwischen Präsident und Nationalversammlung), in: Chin Yŏng-jae (ed.), Hanguk kwollyŏkkujoŭi ihae (Verständnis der Machtstrukturen in Südkorea), Seoul 2004, pp. 295-325; vgl. auch Kang Won-t'aek, Hŏnbŏpkaejŏngŭi chŏngch'i: muŏsŭl pakkul kŏsinga (Die Politik der Verfassungsreform: Was muss geändert werden?), Seoul 2010. Im Unterschied dazu sind einige konservative Politologen der Meinung, man solle sich in Richtung einer dezentralisierten Verfassung, die die lokale Autonomie stärkt, bewegen. Ch'oe Pyŏng-sŏn / Kim Sŏn-hyŏk (eds.), Pun'gwonhŏnbŏp. Sŏnjinhwaro kanŭn kil (Dezentralisierte Verfassung - der Weg zum Fortschritt), Seoul 2007.

33 Yun Tae-kyu, Wae kaehŏninga? (Warum Verfassungsreform?), Seoul 2005; Kim, note 10, pp. 19-39; Sŏng Nak-in, Hŏnbŏpkaejŏng purimgukkaga toeŏsŏnŭn an toenda (Wir dürfen bei der Verfassungsreform kein unfruchtbarer Staat sein), Kosigye 12 (2010), pp. 2-5; Ki Mae-won, Hangukhŏnbŏpsawa hyŏnhaenghŏnbŏp kibon'gwonjangŭi kaejŏngbanghyang (Die koreanische Verfassungsgeschichte und die Richtung der Revision der Grundrechte der gegenwärtigen Verfassung), Kongbŏphakyŏngu 10 (2009) 3, pp. 65-95. Hingewiesen sei auch auf die Koreanische Gesellschaft für Öffentliches Recht, die auf ihren seit 2005 jährlich veranstalteten wissenschaftlichen Konferenzen Fragen der Verfassungsreform unter verschiedenen Blickwinkeln behandelt. 2005 war das Thema der Konferenz „Hŏnbŏpkaejŏng ŏttŏk’e pol kŏsinga“ (Wie sollte die Verfassungsreform gesehen werden?), Kongbŏpyŏngu 34/ 1; 2006 „T'ongilsidaerŭl taebihan hŏnbŏpkaejŏngŭi panghyang“ (Die Richtung der Verfassungsreform in Vorbereitung auf die Wiedervereinigung), Kongbŏpyŏngu 34/ 4 Teil 2; 2008 „Hŏnjŏng 60junyŏn chaengjŏmgwa taean“ (60 Jahre Verfassungsreform - Streitpunkte und Alternativen). Auch die Koreanische Verfassungsgesellschaft gab 2006 den Abschlussbericht der Arbeitsgemeinschaft zur Verfassungsreform „Hŏnbŏpkaejŏngyŏngu“ (Untersuchungen zur Verfassungsreform) heraus und veranstaltete wissenschaftliche Konferenzen, so $2007 \mathrm{zu}$ dem Thema „Realität und Aufgaben des koreanischen Präsidialsystems“ und 2010 unter dem Titel „Detaillierte Untersuchungen zur Notwendigkeit und Richtung der Verfassungsreform - die wünschenswerte Zukunft unserer Verfassung (Hŏnbŏphakyŏngu).

34 Hamkkehanŭn siminhaengdong (ed.), 87nyŏn hŏnbŏp, muŏsi munjeinga. Hŏnbŏp tasi pogi (Die Verfassung von 1987, was ist problematisch an ihr? Ein erneuter Blick auf die Verfassung), Seoul 2007. 
Die Verfassungsdiskussionen, die in der Wissenschaft und der Zivilgesellschaft geführt werden, weisen anders als in der Politik in Bezug auf die Inhalte der einzelnen Postulate eine relative Kontinuität auf. Die Standpunkte unterscheiden sich je nachdem, ob ein Präsidialsystem oder ein Kabinettsystem favorisiert wird, doch wird durchgängig kritisiert, dass die sonstige Verfassungsdiskussion nicht ,der Sicherung der Grundrechte und dem Ausbau der Demokratie dient“, sondern ,ihren Fokus lediglich auf die Revision der Regierungsform richtet". Gleichzeitig wird „die unbekümmerte Illusion, dass eine Änderung der Verfassung die Politik ändern würde“, kritisiert ${ }^{35}$ und festgestellt, dass es „,bei der Erarbeitung der Vorschläge zur Verfassungsänderung ein Verfahren geben sollte, das nach Möglichkeit sämtliche Meinungen, auch Minderheitspositionen, erfasst“". Nicht wenige Stimmen warnen, dass, ,sofern man ohne eine solche Verfahrensweise in Richtung Mehrheitsentscheidung geht, die Gefahr der Entwicklung hin zu einer Willkürherrschaft besteht, die mit den elementarsten Prinzipien von Freiheit im Widerspruch steht. " ${ }^{36}$ Wissenschaftler und zivilgesellschaftliche Akteure machen meist deutlich, warum sie welche Machtstruktur favorisieren.

\section{Politiker}

Im Falle der Verfassungsdiskussionen von Politikern sind die Dinge weitaus komplizierter. Die Standpunkte von Politikern und einzelnen Fraktionen verändern sich mit der politischen Situation. Obwohl sich die Akteure der Diskussionen 2005-2007 kaum von denjenigen unterscheiden, die sich seit 2009 an den Verfassungsdebatten beteiligen, nehmen sie häufig gänzlich gegensätzliche Standpunkte ein.

Präsident Roh Moo-hyun legte während seiner Regierungszeit in einem „Großen Gespräch mit den Bürgern“ einen Ein-Punkt-Plan zur Verfassungsänderung, der eine vierjährige Amtszeit mit einmaliger Verlängerungsmöglichkeit vorsah, vor. Er erklärte dazu: „Ich denke, dass die fünfjährige Amtszeit des Präsidenten, die im Prozess der Verfassungsreform von 1987 mit der Absicht eingeführt wurde, eine langfristige Machtinanspruchnahme institutionell zu verhindern, jetzt ihre Mission erfüllt hat. In der heutigen Wirklichkeit von Südkorea, in der Rechtmäßigkeit und Transparenz bei Wahlen rasch gesichert worden und die demokratischen Kräfte des Volkes gereift sind, ist die Furcht vor einer langfristigen Machtausübung, die der Grund für die Einführung der einmaligen Amtszeit war, bereits überholt. Es ist eher so, dass mittlerweile zahlreiche negative Auswirkungen zutage treten. Die einmalige Amtszeit schadet insbesondere der verantwortungsvollen Ausübung des Präsidentenamtes. Eine Bewertung der Durchführung seiner Amtsgeschäfte bei den nächsten Wahlen ist nicht möglich, und es ist schwer, strategische oder Zukunftsaufgaben des Staates mit Konsequenz und Kon-

35 Song Ki-ch'un, Vortrag, Parlament/Koreanische Verfassungsgesellschaft „Gemeinsame wissenschaftliche Konferenz anlässlich des 50. Jahrestages der Revolution vom 19. April“, Seoul 16.4.2010.

36 Kim Sŏn-hwa, Vortrag, Parlament/Koreanische Verfassungsgesellschaft „Gemeinsame wissenschaftliche Konferenz anlässlich des 50. Jahrestages der Revolution vom 19. April“, Seoul 16.4.2010. 
tinuität voranzubringen. Besonders in der zweiten Hälfte der Amtszeit gestaltet sich eine verantwortungsvolle Durchführung der staatlichen Politik noch schwieriger, sodass im schlimmsten Fall eine staatliche Krise heraufbeschworen werden kann." Er sei der Meinung, „falls die Amtsdauer des Präsidenten von fünf auf vier Jahre bei gleichzeitiger Möglichkeit einer einmaligen Amtsverlängerung korrigiert werde, würde dies entscheidend zur Festigung von Konsequenz und Kontinuität in Bezug auf die strategischen Aufgaben des Staates beitragen.“ Aus diesem Grunde mache er „,en Vorschlag, die Amtszeit des Präsidenten auf vier Jahre mit der Option einer einmaligen Verlängerung und zugleich die Zeitgleichheit mit der gegenwärtigen vierjährigen Legislaturperiode der Abgeordneten der Nationalversammlung festzulegen.“ Ergänzend erklärte er, dass „,unter dem derzeitigen System der fünfjährigen Amtszeit des Präsidenten häufig Wahlen zur Nationalversammlung und zu lokalen Selbstverwaltungsorganen stattfinden. Dies verschärft unnötig politische Konfrontationen und Konflikte, verursacht für die Gesellschaft beträchtliche Kosten und belastet die Stabilität der staatlichen Politik. “37

Seine Begründung der Notwendigkeit einer Verfassungsänderung war logisch. Trotzdem stieß der Vorschlag auf massive Ablehnung seitens der Abgeordneten im Parlament, besonders von der Oppositionspartei GNP. Yi Chae-o, einer der Abgeordneten, der damals energisch seinen Widerstand bekundete, erklärte, er selbst hätte ,nichts gegen den Inhalt der Verfassungsänderung einzuwenden, nur der Zeitpunkt war problematisch. Im Juli jenes Jahres sollte die Wahl des Präsidentschaftskandidaten der GNP stattfinden. Nur sechs Monate vorher wollte man eine Verfassungsreform durchführen, die die ganze Wahl durcheinander gebracht hätte. Wie also hätte man einen solchen Vorschlag annehmen können?“38 Dass es nicht nur eine Frage des richtigen Timings war, macht ein offener Brief von ihm an Präsident Roh Moohyun, in dem er dessen Vorschlag der Bildung einer Großen Koalition ablehnt, deutlich. In diesem Brief, den er am 3.9.2005 unter dem Titel „Präsident Roh Moo-hyun, beklommen fühlt sich das Volk“ auf die Homepage der GNP stellte, fordert er diesen zum Rücktritt auf: „Eine Änderung der Verfassung, in welcher Form auch immer, kann es während Ihrer Amtszeit nicht geben. ... Wenn Ihnen alles zur Last wird und Sie kein Selbstvertrauen mehr haben, dann ziehen Sie sich einfach still und leise zurück. “" ${ }^{39}$ Unter Lee Myung-bak wurde Yi Chae-o später zu einem der eifrigsten Befürworter einer Verfassungsreform.

Solche Positionswechsel sind auch in der gegenwärtig stattfindenden Verfassungsdebatte eine häufig zu beobachtende Erscheinung. Auch Präsident Lee Myung-bak selbst, der das Parlament seit 2009 häufig aufforderte, über eine Verfassungsreform zu beraten, hat einen derartigen Wechsel vollzogen. In einem Interview mit der Zeitung Hankyoreh vom 25.1.2006 hatte er Präsident Rohs, dem damals noch zwei Jahre im Amt verblieben, mit den Worten

37 Roh Moo-hyun, Kungminyŏrŏbunkke tŭrinŭn malssŭm. Ch'aeg'iminnŭn kukchŏngŭl wihae 4nyŏn yŏnim kaehŏn chean (Worte an die Bürger. Vorschlag zur Änderung der Verfassung in eine vierjährige verlängerbare Amtsperiode für eine verantwortungsvolle Politik), 9.1.2007.

38 Yijaeo int‘ŏbyu (Interview mit Yi Chae-o), Chugandonga (Weekly Dong-a), 21.2.2010.

$39 \mathrm{http}: / / w w w . v i e w s n n e w s . c o m /$ article/view.jsp?seq=72003. 
verurteilt: „Jede einzelne Partei sollte einen guten Plan zur Verfassungsänderung als öffentliches Versprechen aufstellen und den Bürgern vorlegen, und die gewählte Person sollte diesen dann innerhalb von ein, zwei Jahren in einem sorgfältigen Verfahren umsetzen. Es geht nicht an, dass eine Regierung zu ihrem eigenen Vorteil die Verfassung ändert.“ Das hinderte ihn nicht, ab Februar 2011, als er selbst noch zwei Jahre Amtszeit vor sich hatte, das Parlament wiederholt zu drängen, die Verfassungsreform auf die Tagesordnung zu setzen.

Die Verfassungsdebatte in der Regierungszeit von Lee Myung-bak zeigt ein anderes Bild als unter Roh Moo-hyun. Im Unterschied zu Präsident Roh Moo-hyun fordert Präsident Lee Myung-bak lediglich zur Diskussion auf, vermeidet jedoch konkrete Richtungsaussagen. Diese Aufgabe überlässt er einer ihn unterstützenden Gruppe innerhalb der Regierungspartei GNP und dem von ihm als Minister für besondere Aufgaben ernannten Yi Chae-o. Sie schlagen kein Präsidialsystem mit vierjähriger Amtsperiode und der Möglichkeit einer Wiederwahl, sondern ein semipräsidentielles Regierungssystem, in dem der Präsident nur für die Außen- und Verteidigungspolitik und ein vom Parlament gewählter Ministerpräsident für die Innenpolitik zuständig sind, vor. Der Vorsitzende der GNP An Sang-su begründet dies damit, dass „,ie Forderung nach einer Dezentralisierung der Macht eine zeitgemäße Forderung und eine Position ist, die auch im Parlament gründlich diskutiert werden sollte. “40 Doch bezüglich der Frage, welche konkrete Form dieses semipräsidentielle Regierungssystem annehmen soll, unterscheiden sich die Standpunkte des Präsidenten und des von ihm ernannten Sonderministers. Minister Yi Chae-o äußerte, dass „ein machtgeteiltes Präsidialsystem koreanischer Prägung mit vierjähriger verlängerbarer Amtszeit" erstrebenswert sei. Vom Präsidenten wurde berichtet, dass er bei einem gemeinsamen Frühstück mit der Führung der GNP am 30.11.2009 die gegenwärtige einmalige Amtszeit von fünf Jahren favorisierte, indem er sagte: „Ich denke, die Option der Amtsverlängerung könnte das Arbeiten aus Überzeugung erschweren, weil man mit der Wiederwahl im Kopf diese und jene Rücksichten nehmen muss. ... Meine bisherige Tätigkeit hat mir gezeigt, dass eine einmalige Amtszeit für ein verantwortungsvolles Arbeiten besser zu sein scheint."41 In solchen Inkongruenzen, die an Beliebigkeit grenzen, liegt ein wichtiger Grund die Existenz mannigfaltiger Narrative, aus denen sich letztlich kein spezifischer Diskursverbund herausbilden kann. Dazu gehört auch, dass Politiker ihre Standpunkte zur Verfassungsreform aus Opportunitätsgründen und mit dem politischen Kontext verändern.

Seit 2009 sind die Reaktionen der Abgeordneten der Demokratischen Partei denen der GNP-Abgeordneten 2007 ganz ähnlich. Auch sie sagen nun, die ganze Diskussion diene lediglich „der Politikwende“. ${ }^{42}$ „Für die Pro-LMB-Fraktion scheint die Vorstellung, dass die ehemalige Parteivorsitzende Park (Park Geun-hye, Anm. d. Ü.) unter dem gegenwärtigen Verfassungssystem Präsidentin werden könnte, eine gewaltige politische Belastung zu sein, ja regelrechte Furcht auszulösen. ... Wahrscheinlich denken sie, dass sie, wenn sie durch eine

40 Gemeinsame Sitzung der obersten Parteiführer der GNP, Hankyoreh, 16.9.2009.

41 http://www.viewsnnews.com/article/view.jsp?seq=72003.

42 Chŏng Se-gyun, Rede auf der Vorstandssitzung der Demokratischen Partei, Hankyoreh, 16.9.2009. 
eigene Verfassungsänderung eine Machtteilung erreichten, wenigstens den Ministerpräsidenten stellen könnten, um schließlich an der Macht teilhaben ... zu können. “43 Diese Aussage des früheren Fraktionsvorsitzenden der Demokratischen Partei Yi Kang-nae verdeutlicht das Misstrauen, das zwischen Parteien und selbst zwischen parteiinternen Gruppierungen herrschen kann.

In dieser Situation hochgradigen Misstrauens ist eine demokratische Konsensfindung nicht zu erzielen. Auch der gegenwärtig in Korea ablaufende Diskurs zur Verfassungsreform erfolgt nicht im Dialog, sondern durch zeitversetzt vonstatten gehende Monologe. Man redet nicht miteinander, weil man meint, dass eine Verfassungsänderung durch eine politische Entscheidung im Sinne von Carl Schmitt zustande kommen muss. Dafür sucht man im besten Fall nach einer überzeugenden Begründung.

Ein Grund, warum viele Politiker heutzutage eine Revision der Verfassung befürworten, ist der, dass „eine neue Verfassung notwendig ist, wenn man zu einer führenden Nation aufsteigen will.“" ${ }^{44}$ Dass eine solche Argumentation keine Überzeugungskraft besitzt, machte ein führender Abgeordneter der GNP, Hong Chun-p’yo, deutlich: „Die japanische Verfassung wurde nach Kriegsende 1946 verabschiedet und ist seit dem kein einziges Mal revidiert worden. Mit dieser Verfassung hat sich Japan zu einem führenden Land entwickelt." Gleichzeitig betonte er, „wenn das Parlament eine Änderung der Verfassung will, muss dem Verlangen des Volkes entsprochen werden“, und fuhr fort: „Betrachtet man frühere Verfassungsreformen, bei denen das Parlament die Initiative ergriffen hatte, war es so, dass ihm bei der 3. und 4. Revision wegen der Umstände des Rücktritts des Präsidenten nach der Revolution vom 19. April, gar nichts anderes übrig blieb. Die 6. Änderung wurde zwar vom Parlament durchgeführt, aber in Wirklichkeit hatte eine der Regierung hörige Volksvertretung deren Rücktritt angenommen und daraufhin die dreimalige Wiederwahl beschlossen; der Wille des Volkes war gar nicht berücksichtigt worden. Die letzte Revision wurde vom Parlament aus eigenem Antrieb durchgeführt, und zwar auf der Grundlage des Begehrens der Bürger im Zuge der Widerstandsbewegung vom Juni 1987. ... Es war nur zwei Mal der Fall, dass das Parlament aus sich heraus dem Willen des Volkes folgend eine Verfassungsänderung vorgenommen hat.“ Und warf die Frage auf, „ob denn jetzt der Wille des Volkes vorhanden sei, der das Parlament in die Lage versetze, aus freien Stücken eine Verfassungsänderung zu initiieren. “45

In der Tat spielen die Bürger, die gemäß der gegenwärtig gültigen Verfassung das Recht haben, durch eine Volksabstimmung eine abschließende Entscheidung zur Verfassungsän-

43 P'ŭresian (Pressian), 25.2.2010.

44 In seiner Festrede 2010 zum Tag der Befreiung sagte Präsident Lee Myung-bak, dass „für den politischen Fortschritt eine Verfassungsänderung “ durchgeführt werden müsse (Yi Chae-o, Interview mit Hankyoreh 16.2.2011). Als Fraktionsvorsitzender forderte Kim Mu-sŏng am 21.2.2011 im Parlament: „Lassen Sie uns die Verfassung eines entwickelten Landes erarbeiten, die den Bedingungen des 21. Jahrhunderts entspricht.“ Das in allen Bereichen Koreas - Politik, Gesellschaft, Wirtschaft und Kultur -zu beobachtende beharrliche Festhalten an dem Terminus ,führende Nation, entwickeltes/fortschrittliches Land“" (sŏnjinguk) sollte unbedingt einer genaueren Analyse unterzogen werden.

Hong Chun-p'yo, Rede auf der Vorstandssitzung der GNP, OhmyNews, 14.2.2011. 
derung zu fällen, in der derzeitigen Diskussion um dieses Thema keine wichtige Rolle. Auch Präsident Roh, der 2007 durch das „Große Gespräch mit den Bürgern“ seinen Vorschlag zur Verfassungsreform unterbreitet und sich dabei alle Mühe gegeben hatte, das Volk, den Souverän und obersten Entscheider in dieser Angelegenheit, zu überzeugen, sagte damals, er werde, ,falls die Revision nicht zustande kommt, diejenigen, die dagegen waren, unablässig auf ihre Verantwortung hinweisen." Gleichzeitig hatte er seinen Willen zum Ausdruck gebracht, aus innerer Überzeugung heraus die Verfassungsänderung vorzuschlagen, da er nicht warten könne, „bis sich die öffentliche Meinung umkehrt.“46 Auch hier ist das Volk nicht Akteur, sondern bestenfalls ein Akteur, der erst noch überzeugt werden muss.

Ähnliche Erscheinungen lassen sich auch in der Debatte seit 2009 beobachten. Der Fraktionsvorsitzende der GNP Kim Mu-sŏng hatte am 16.9.2009, als er noch nicht der innerparteilichen Gruppe für eine Verfassungsänderung, die den Präsidenten unterstützte, angehörte, die Ansicht vertreten, dass ,eine Diskussion um die Revision der Verfassung, die nur politische Standpunkte widerspiegelt, nur schwerlich Unterstützung erfahren wird; nur ein Sympathiebündnis der Bürger kann den erforderlichen Antrieb geben. “47 Dass er dann am 21.2.2011 in der Nationalversammlung die Gründung eines Sonderausschusses zur Verfassungsreform vorschlug, lag ganz sicher nicht daran, dass sich ein Sympathiebündnis der Bürger formiert hätte. Die seit 2005 zu diesem Thema erfolgenden Meinungsumfragen zeigen vielmehr, dass immer weniger Bürger eine Verfassungsreform für notwendig halten. Wenn überhaupt sollte diese nicht unter der derzeitigen, sondern erst unter der nächsten Regierung angestrebt werden. Je weiter die zweite Hälfte der Amtszeit voranschreitet, desto mehr gewinnt diese Meinung an Gewicht. Zudem bleibt die Unterstützung eines Präsidialsystem mit vierjähriger Amtszeit und Wiederwahlmöglichkeit gegenüber einer fünfjährigen einmaligen Amtszeit im Zeitablauf unverändert (s. Anhang 1). Dass dessen ungeachtet Politiker, die ein semipräsidentielles Regierungssystem verteidigen, konkrete Zeiträume für eine Verfassungsreform festlegen und erklären, diese bis dahin abzuschließen, ${ }^{48}$ bedeutet, dass sie die Meinung ihres Wahlvolkes, das das ultimative Recht zur Verfassungsänderung besitzt, nicht berücksichtigen.

46 Hankyoreh, 18.1.2007.

47 Meldung der Hankyoreh, 16.9.2009.

48 So erklärte der ehemalige Fraktionsvorsitzende der GNP An Sang-su im September 2009 öffentlich, dass er, „sobald der Sonderausschuss des Parlaments zur Verfassungsreform nach den Nachwahlen im Oktober gegründet worden ist, dafür sorgen wird, dass die Frage der Machtstruktur vorrangig und abschließend in der ersten Hälfte des kommenden Jahres behandelt wird.“ (Hankyoreh, 16.9.2009) Die meisten Meinungsumfragen 2005-07 ergaben eine absolute Mehrheit zugunsten einer Verfassungsänderung und eine einfache Mehrheit für eine Umsetzung durch die nächste Regierung (s. Anhang 1). In den Umfragen 2011 gewann die Ablehnung der Verfassungsreform leicht die Oberhand. Unter denjenigen, die ihr generell zustimmten, überwog wiederum die Zahl derjenigen, die sie der nächsten Regierung übertragen wollten. 


\section{Medien}

Die Medien, vor allem die Zeitungen, spielen eine wichtige Rolle im Diskurs um die Verfassungsreform. Auch ihre Standpunkte haben sich wie bei den Politikern mit dem politischen Kontext verändert. So konnte sich auch über die Medien kein Diskursverbund um ein dominierendes Narrativ herausbilden.

Wie das Thema des Leitartikels in der Segye Ilbo vom 18.10.2010 „Diskussion um Verfassungsreform ohne Zustimmung der Bürger birgt Gefahr politischer Ränke“ zeigt, versäumen die Medien nicht, auf die Probleme einer Debatte hinzuweisen, bei der die Meinung des Volkes, des Souveräns, außen vor bleibt. Dennoch sind sie, wie die Untersuchung von Leitartikeln mehrerer Zeitungen ergab, nicht so sehr daran interessiert, die Schwachpunkte der von der Politik geführten Debatte aufzuzeigen, sondern versuchen vielmehr, mit einer eigenen bestimmten Parteilichkeit die Öffentlichkeit zu beeinflussen. ${ }^{49}$ Beispielsweise kritisiert die konservative Chosun Ilbo die Vorschläge von Roh Moo-hyun zur Verfassungsreform auf das Heftigste. Zwischen dem 9.1.2007, dem Tag des „Großen Gesprächs mit den Bürgern“, und dem 15.4.2007, als er seinen Vorschlag wieder zurückzog, veröffentlichte sie 13 Leitartikel, die sich allesamt gegen diese Verfassungsreform aussprachen. ${ }^{50} \mathrm{Im}$ Gegensatz dazu wurde zwischen dem 15.8.2009 und dem 25.3.2011, in dem Präsident Lee mehrfach das Parlament aufforderte, in die Diskussion über Verfassungsänderung einzutreten, dieses Thema in den Leitartikeln der Chosun Ilbo acht Mal behandelt. Deren Tenor war freundlich, und dem Präsidenten wurden Ratschläge erteilt. Während 2007 über Kritik und Widerstand ausführlich und oft berichtet wurde, ${ }^{51}$ findet sich kaum ein Artikel, der konkrete Informationen über Motive und Inhalte des Vorschlags Präsident Rohs liefert. ${ }^{52}$ Im Gegensatz zur Chosun Ilbo brachte die zum fortschrittlichen Lager gehörende Hankyoreh Sinmun Informationen analytischen

49 Die Parteilichkeit der „Chojungdong“ (= Chosun Ilbo, Chungang Ilbo und Dong-a Ilbo) genannten wichtigsten Zeitungen ist sprichwörtlich und häufig untersucht worden.

50 In diesem Zeitraum fordert die Chosun Ilbo vom Präsidenten, ,nicht von Verfassungsänderung, sondern über eine weitsichtige Politik des Staates zu reden“ (12.1.2007) und fragt, „,womit er wohl als nächstes die Welt erschüttern wolle“(11.1.2007). Sie verurteilt „die Regierung, die ihr Werbematerial für eine Verfassungsänderung in Zeitungen in die Wohnzimmer schmuggelt" (26.3.2007) und fordert, das für die Verfassungsreform Werbung machende „Informationsamt der Regierung ins Museum zu schicken“(28.3.2007). „Das Volk unterstützt einen Präsidenten, der seines Amtes würdig ist“"schreibt die Chosun Ilbo am 5.4.2007 und will damit die Aussage des Präsidenten, ins Parlament zu gehen um den von ihm vorgelegten Vorschlag zur Revision der Verfassung zu erläutern, verspotten. An anderer Stelle: „Hält der Präsident in seinem Starrsinn an der Verfassungsreform fest, weil er nicht gern in die Knie geht?" (13.4.2007).

51 Vom 9.1.2007 bis 15.4.2007 erschienen in der Chosun Ilbo insgesamt 199 Artikel, bei denen in der Überschrift und im Text das Wort „Kaehŏn“ (Verfassungsreform) vorkam. Meist soll ein negatives Image dieser Initiative vermittelt werden. http://srchdb1.chosun.com/pdf/i_archive/search.jsp.

52 Zwischen dem 15.8.2009 und dem 25.3.2011 erschienen in der Chosun Ilbo insgesamt 253 Artikel, in denen das Wort „Kaehŏn“ (Verfassungsreform) vorkam. Die meisten von ihnen waren keine analytischen Beiträge, die konkrete Informationen zu dem Thema lieferten, sondern übermittelten ungefiltert die Standpunkte von Politikern der Regierungspartei. http://srchdb1.chosun.com/pdf/i_archive/search.jsp. 
Charakters zu diesem Vorschlag. ${ }^{53}$ Dennoch unterscheidet sich die Bewertung der beiden Zeitungen in den beiden Zeiträumen (9.1 bis 15.4.2007 sowie 9.1.2009 bis 25.3.2011), wenn man sie mit umgekehrten Vorzeichen liest, kaum. ${ }^{54}$ Hankyoreh ist 2007 Präsident Roh eher freundlich gesonnen und gibt auch wohlgemeinte kritische Ratschläge, während sie später Präsident Lee und der Regierungspartei vorwirft, nur aus politischem Kalkül zu handeln. ${ }^{55}$ Der Tenor der Leitartikel beider Zeitungen ähnelt sich dermaßen, dass man sie nicht unterscheiden kann, wenn man die Namen der Präsidenten und das Datum der Veröffentlichung streicht. ${ }^{56}$

\section{Schlussbemerkungen}

Infolge der inhärenten Besonderheiten der Diskurse um die Verfassungsreform kommt es zu keinen diesbezüglichen politischen Entscheidungen. Allerdings verdeutlicht der von Politikern getragene Diskurs deren Einstellung zur Verfassung und zum Volk als Souverän. Die derzeit gültige Verfassung ist eine rigide Verfassung, in der das Volk als verfassungsgebende Gewalt und letzte Instanz einer Verfassungsänderung genannt ist. Inwieweit diese Bestimmungen der Verfassung ihre Gültigkeit entfalten und in welchem Maße das Volk als Souverän und die Autorität der Verfassung geachtet werden, ist ein anderes Problem und unabhängig von der Verankerung im Verfassungstext.

Seit die Volksabstimmung 1962 als höchste Instanz für Verfassungsänderungen eingeführt wurde, hat es keinen Fall einer Ablehnung durch das Volk gegeben. Selbst als ein Diktator, der die demokratischen Sehnsüchte des Volkes mit Füßen trat, zum Zwecke des eigenen Machterhalts eine Verfassungsreform einbrachte, wurde diese in einer Volksabstimmung angenommen. In diesem Sinne ist die Revision von 1987 in der koreanischen Verfassungsge-

53 In der Hankyoreh Sinmun erschienen vom 9.1.-15.4.2007 insgesamt 181 Artikel, in denen das Wort „Kaehŏn“(Verfassungsreform) vorkam. Darunter waren auch vier große Beiträge, die die konkreten Inhalte des Vorschlags kritisch und präzise analysierten und in Form von Pro und Kontra gegenüber stellten (10.1.2007, 16.3.2007, 9.3.2007). Hingegen wurde vom 15.8.2009-25.3.2011 in 310 Artikeln „Kaehŏn“ erwähnt. Größere Beiträge wie 2007 waren nicht darunter. http://www.kinds.or.kr/.

54 Während der beiden Perioden behandelt Hankyoreh die Verfassungsreform jeweils in 12 Leitartikeln.

55 In ihren Leitartikeln 2007 kritisierte Hankyoreh Präsident Roh, der gesagt hatte, aus seiner Überzeugung heraus die Initiative zur Verfassungsänderung zu ergreifen: „Das Interesse der Nation hat Vorrang vor der Überzeugung des Präsidenten“ (30.3.2007) und „Debatte um Verfassungsreform ohne Unterstützung durch die öffentliche Meinung nichts als graue Theorie“ (12.1.2007). An die GNP gerichtet kritisierte sie, diese solle die Diskussion um eine Verfassungsreform nicht immer nur ablehnen, sondern „Alternativen und eine verantwortungsbewusste Haltung“ zeigen (27.1.2007). Zur Debatte 2009-2011 dagegen merkt sie an, dass der Präsident nur Feuer anzünde und politisches Chaos entfache; der „Einsatz der Verfassung für die Cliquenpolitik“ dürfe nicht gestattet werden. Die Parteikonferenz der GNP zur Verfassungsreform bezeichnet sie lediglich als „politisches Spielchen“, das „nur den Verdruss der Bürger schürt“, und stellt die vorwurfsvolle Frage, ob die GNP und der Präsident „die Bürger zum Narren halten oder bedrohen“ (11.12.2010).

56 Der Ton in den Leitartikeln der Hankyoreh-Zeitung ist lediglich weniger vulgär als der der Chosun Ilbo. 
schichte ein epochales Ereignis, wurde sie doch im Widerstand gegen den Diktator Chun Doohwan, der eine Änderung der auf der indirekten Wahl des Präsidenten basierenden Verfassung von 1981 ablehnte, und unter großen Opfern des Volkes erreicht. Somit hatte das Volk als Souverän damals zum ersten Mal mit verfassungsgebender und verfassungsrevidierender Gewalt seine tatsächliche Stärke unter Beweis gestellt. Nach 1987 kam es letztendlich nur noch darauf an, die Unterstützung des Volkes für eine Verfassungsreform zu gewinnen.

Interessant an Verfassungsdebatten der letzten Jahre ist, dass sie gar nicht in die Richtung der Bildung eines nationalen Konsenses gehen. Dass man die Notwendigkeit der Revision gar nicht erläutert, sondern nur festlegt, bis wann sie abgeschlossen sein sollten, liegt daran, dass man die Zustimmung in einem Volksentscheid als selbstverständlich voraussetzt. Das belegt, dass im Bewusstsein der politischen Elite Koreas durchaus noch autoritäre Denkansätze vorhanden sind.

Während der Chosŏn-Dynastie und in der Zeit des Autoritarismus hatte das Volk die Pflicht, sich den von den Machthabern beschlossenen Gesetzen zu fügen. Auch in der demokratisierten koreanischen Gesellschaft genießen Politiker als politische Entscheidungsträger große Autorität. Hohe Autorität darf kein autoritäres Denken rechtfertigen. Noch weniger darf man zulassen, dass sich autoritäres Denken direkt in der Einstellung zur Verfassung widerspiegelt. Wenn sich, wie in Korea, solches autoritäres Denken mit Schmittschen Anschauungen von Politik und Verfassung verbindet, kann auch die Demokratie selbst in Gefahr geraten. Aus diesem Grunde ist es geboten, die sich auch in der Gegenwart fortsetzenden Verfassungsdiskurse wachsam und genau zu beobachten. 
Anhang - Meinungsumfragen zur Verfassungsreform 2005-2011

\begin{tabular}{|c|c|c|c|c|}
\hline \multirow{2}{*}{$\begin{array}{r}\text { Umfrageinstitut/ } \\
\text { Auftraggeber }\end{array}$} & \multirow{2}{*}{$\begin{array}{l}\text { Unter- } \\
\text { suchungs- } \\
\text { zeitraum }\end{array}$} & \multicolumn{3}{|c|}{ Ergebnisse } \\
\hline & & $\begin{array}{c}\text { Pro und Kontra } \\
\text { Verfassungsreform }\end{array}$ & $\begin{array}{c}\text { Zeitpunkt der } \\
\text { Verfassungsreform }\end{array}$ & Machtform \\
\hline $\begin{array}{l}\text { Korea Society Opi- } \\
\text { nion Institute } \\
\text { (KSOI)/ Kookmin } \\
\text { Ilbo }\end{array}$ & 21.2.2011 & $\begin{array}{l}\text { ja: } 54,8 \% \\
\text { nein: } 37,3 \% \\
\text { Enthaltung: } 7,9 \%\end{array}$ & $\begin{array}{l}\text { nächste Reg.: } 49,2 \% \\
\text { jetzige Reg.: } 41,4 \% \\
\text { Enthaltung: } 9,4 \%\end{array}$ & \\
\hline Hankyung & 20.2 .2011 & $\begin{array}{l}\text { ja: } 41,1 \% \\
\text { nein: } 23,9 \% \\
\text { Enthaltung: } 35,0 \%\end{array}$ & $\begin{array}{l}\text { nächste Reg.: } 43,1 \% \\
\text { jetzige Reg.: } 41,4 \% \\
\text { Enthaltung: } 9,4 \%\end{array}$ & \\
\hline Dongseo Research & 11.1 .2011 & & $\begin{array}{l}\text { jetzige Reg.: } 34,8 \% \\
\text { nächste Reg.: } 35 \% \\
\text { nach der nächsten Re- } \\
\text { gierung: } 18 \%\end{array}$ & $\begin{array}{l}\text { Beibehaltung der ein- } \\
\text { maligen Amtszeit: } \\
43 \% \\
\text { Präsidialsystem, } 4 \\
\text { Jahre, Wiederwahl: } \\
32 \% \\
\text { semipräs. System: } \\
8,3 \% \\
\text { Kabinettsystem: } 7,0 \%\end{array}$ \\
\hline Seoul Shinmun & 3.1 .2011 & $\begin{array}{l}\text { ja: } 39,3 \% \\
\text { nein: } 57,3 \%\end{array}$ & & $\begin{array}{l}\text { bei Revision } 4 \text { Jahre, } \\
\text { Wiederwahl: } 45,3 \%\end{array}$ \\
\hline Dong-a Ilbo & 3.1 .2011 & $\begin{array}{l}\text { nein: } 37,9 \% \\
\text { ja: } 23,9 \%\end{array}$ & $\begin{array}{l}\text { ja unter der nächsten } \\
\text { Regierung: } 29,2 \%\end{array}$ & \\
\hline $\begin{array}{l}\text { KM Institute/The } \\
\text { Herald Business }\end{array}$ & 3.1 .2011 & $\begin{array}{l}\text { nicht notw.: } 26,6 \% \\
\text { ja: } 32,1 \% \\
\text { zur Amtszeit der ge- } \\
\text { genwärtigen Regie- } \\
\text { rung } \\
\text { nein: } 26,4 \%\end{array}$ & & $\begin{array}{l}\text { jetzige einmal. Amts- } \\
\text { zeit von } 5 \text { Jahren: } \\
28,6 \% \\
\text { Präsidialsystem, } 4 \\
\text { Jahre, Wiederwahl: } \\
33,8 \% \\
\text { semipräs. System: } \\
11,8 \% \\
\text { Kabinettsystem: } \\
11,5 \%\end{array}$ \\
\hline KBS & 1.1 .2011 & notw.: $69,4 \%$ & & \\
\hline $\mathrm{MBC}$ & 1.1 .2011 & ja: $44,3 \%$ & & \\
\hline $\begin{array}{l}\text { Real Meter/The } \\
\text { Asia Economy Dai- } \\
\text { ly }\end{array}$ & $23 .-24.12 .2010$ & $\begin{array}{l}\text { ja: } 61 \% \\
\text { nicht notw.: } 22,3 \% \\
\text { Enthaltung: } 16,7 \%\end{array}$ & $\begin{array}{l}\text { nächste Reg.: } 30,8 \% \\
\text { jetzige Reg.: } 30,2 \%\end{array}$ & \\
\hline $\begin{array}{l}\text { Media Research/ } \\
\text { Korea Times }\end{array}$ & $25 .-26.12 .2010$ & $\begin{array}{l}\text { ja: } 39,1 \% \\
\text { nein: } 22,9 \% \\
\text { weiß nicht: } 38,0 \% \\
\text { umfass. Rev. } 61,5 \% \\
\text { Anderung der einma- } \\
\text { ligen Amtszeit } 31,5 \%\end{array}$ & & $\begin{array}{l}\text { Gegenwärtiges Präsi- } \\
\text { dialsystem: } 36,3 \% \\
\text { Präsidialsystem, } 4 \\
\text { Jahre mit Wieder- } \\
\text { wahl: } 33,7 \% \\
\text { semipräs.System: } \\
\text { 12,4\% } \\
\text { Kabinettsystem: } 4,2 \%\end{array}$ \\
\hline
\end{tabular}




\begin{tabular}{|c|c|c|c|c|}
\hline \multirow{2}{*}{$\begin{array}{r}\text { Umfrageinstitut/ } \\
\text { Auftraggeber }\end{array}$} & \multirow{2}{*}{$\begin{array}{c}\text { Unter- } \\
\text { suchungs- } \\
\text { zeitraum }\end{array}$} & \multicolumn{3}{|c|}{ Ergebnisse } \\
\hline & & $\begin{array}{l}\text { Pro und Kontra } \\
\text { Verfassungsreform }\end{array}$ & $\begin{array}{c}\text { Zeitpunkt der } \\
\text { Verfassungsreform }\end{array}$ & Machtform \\
\hline $\begin{array}{l}\text { KM Institute/The } \\
\text { Herald Business }\end{array}$ & $24 .-25.9 .2010$ & $\begin{array}{l}\text { ja: } 63,7 \% \\
\text { umfass. Rev. } 22,0 \% \\
\text { beschränkte } \\
\text { Rev. } 41,7 \% \\
\text { nein: } 20,5 \%\end{array}$ & & \\
\hline Youido Institute & $\begin{array}{l}26.8 .2010 \\
(3.9 .2010)\end{array}$ & ja: $75 \%$ & bis $2010: 58,8 \%$ & $\begin{array}{l}\text { Präsidialsystem, } 4 \\
\text { Jahre, Wiederwahl: } \\
48,2 \% \\
(42,9 \%) \\
\text { semipräs. System: } \\
35,2 \% \\
(44,6 \%) \\
\text { Kabinettsystem: } \\
10,4 \%\end{array}$ \\
\hline $\begin{array}{l}\text { The Herald Busi- } \\
\text { ness }\end{array}$ & 29.3.2010 & & & $\begin{array}{l}\text { Präsidialsystem, } 4 \\
\text { Jahre, Wiederwahl: } \\
38,7 \% \\
\text { Beibehaltung der } \\
\text { einmaligen Amtszeit } \\
\text { von 5 Jahren: } 29,4 \% \\
\text { Semipräs. System: } \\
14,6 \% \\
\text { Kabinettsystem: } \\
10,8 \%\end{array}$ \\
\hline $\begin{array}{l}\text { Media Research/ } \\
\text { Hankook Ilbo }\end{array}$ & 21.9 .2009 & & $\begin{array}{l}\text { vor Lokalwahlen im } \\
\text { Juni 2010: } 28,0 \% \\
\text { vor Präsidentschafts- } \\
\text { wahl 2012: } 37,2 \% \\
\text { nächste Reg.: } 22,6 \% \\
\text { weiß nicht: } 12,2 \%\end{array}$ & $\begin{array}{l}\text { Präsidialsystem, } 4 \\
\text { Jahre mit Wieder- } \\
\text { wahl: } 36,0 \% \\
\text { Beibehaltung der } \\
\text { einmaligen Amtszeit } \\
\text { von } 5 \text { Jahren: } 30,6 \% \\
\text { Semipräs. System: } \\
16,2 \% \\
\text { Kabinettsystem: } 9,3 \%\end{array}$ \\
\hline $\begin{array}{l}\text { Media Research/Si- } \\
\text { sa Journal }\end{array}$ & 8.7.2009 & ja: $62,1 \%$ & $\begin{array}{l}\text { zu den Lokalwahlen } \\
\text { 2010: } 33,1 \% \\
\text { jetzt sofort: } 22,6 \% \\
\text { nächste Reg.: } 15,9 \%\end{array}$ & $\begin{array}{l}\text { Präsidialsystem, } 4 \\
\text { Jahre mit Wieder- } \\
\text { wahl: } 44,9 \% \\
\text { einmal. Amtszeit: } \\
34,5 \% \\
\text { Kabinettsystem: } \\
10,0 \% \\
\text { Semipräs. System: } \\
5,7 \%\end{array}$ \\
\hline $\begin{array}{l}\text { Media Research/ } \\
\text { Hankook Ilbo }\end{array}$ & 9.6 .2009 & & & $\begin{array}{l}\text { Präsidialsystem, } 4 \\
\text { Jahre mit Wieder- } \\
\text { wahl: } 40,9 \% \\
\text { einmal. Amtszeit } \\
\text { von } 5 \text { Jahren: } 29,4 \% \\
\text { Kabinettsystem: } \\
13,4 \% \\
\text { Semipräs. System: } \\
4,1 \%\end{array}$ \\
\hline
\end{tabular}




\begin{tabular}{|c|c|c|c|c|}
\hline \multirow{2}{*}{$\begin{array}{c}\text { Umfrageinstitut/ } \\
\text { Auftraggeber }\end{array}$} & \multirow{2}{*}{$\begin{array}{l}\text { Unter- } \\
\text { suchungs- } \\
\text { zeitraum }\end{array}$} & \multicolumn{3}{|c|}{ Ergebnisse } \\
\hline & & $\begin{array}{c}\text { Pro und Kontra } \\
\text { Verfassungsreform }\end{array}$ & $\begin{array}{c}\text { Zeitpunkt der } \\
\text { Verfassungsreform }\end{array}$ & Machtform \\
\hline $\begin{array}{l}\text { TNS/Büro des Pre- } \\
\text { mierministers }\end{array}$ & 24.3 .2007 & $\begin{array}{l}\text { ja: } 71,4 \% \\
\text { nein: } 25,6 \%\end{array}$ & & $\begin{array}{l}\text { Präsidialsystem, } 4 \\
\text { Jahre mit Wieder- } \\
\text { wahl: } 55,8 \% \\
\text { Präsidialsystem, } 5 \\
\text { Jahre, einmal. Amts- } \\
\text { zeit: } 41,0 \%\end{array}$ \\
\hline $\begin{array}{l}\text { Research and Re- } \\
\text { search /Segye Ilbo }\end{array}$ & 27.1 .2007 & $\begin{array}{l}\text { 4-jährige wiederhol- } \\
\text { bare Amtszeit' } \\
\text { ja: } 47,0 \% \\
\text { nein: } 41,9 \%\end{array}$ & $\begin{array}{l}\text { nächste Reg.: } 56,5 \% \\
\text { zur Amtszeit von Prä- } \\
\text { sident Roh: } 23,1 \%\end{array}$ & \\
\hline $\begin{array}{l}\text { SBS/Hankook Re- } \\
\text { search }\end{array}$ & 9.1 .2007 & & $\begin{array}{l}\text { nächste Reg.: } 55,2 \% \\
\text { jetzige Reg.: } 24,8 \%\end{array}$ & $\begin{array}{l}\text { Präsidialsystem, } 4 \\
\text { Jahre mit Wieder- } \\
\text { wahl: } \\
\text { ja: } 48,4 \% \\
\text { nein: } 42,6 \%\end{array}$ \\
\hline $\begin{array}{l}\text { MBC/Korea Re- } \\
\text { search }\end{array}$ & 9.1 .2007 & & $\begin{array}{l}\text { nächste Reg.: } 63 \% \\
\text { jetzige Reg.: } 29 \%\end{array}$ & $\begin{array}{l}\text { Präsidialsystem, } 4 \\
\text { Jahre mit Wiederwahl } \\
\text { ja: } 51 \% \\
\text { nein: } 40 \%\end{array}$ \\
\hline $\begin{array}{l}\text { KBS/Media Re- } \\
\text { search }\end{array}$ & 9.1 .2007 & & & $\begin{array}{l}\text { Präsidialsystem, } 4 \\
\text { Jahre mit Wiederwahl } \\
\text { unter der gegenwärti- } \\
\text { gen Regierung } \\
\text { ja: } 52,9 \% \\
\text { nein: } 47,1 \%\end{array}$ \\
\hline $\begin{array}{l}\text { Hankook Research/ } \\
\text { Munhwa Ilbo }\end{array}$ & $29 .-30.10 .2005$ & $\begin{array}{l}\text { notw.: } 65,0 \% \\
\text { nicht notw.: } 34,5 \%\end{array}$ & $\begin{array}{l}\text { nächste Reg.: } 41,7 \% \\
\text { bei Präsidentschafts- } \\
\text { wahl } 2007: 21,5 \% \\
\text { bei den Lokalwahlen } \\
\text { Mai 2006: } 19,3 \% \\
\text { jetzt sofort: } 16,3 \%\end{array}$ & $\begin{array}{l}\text { Präsidialsystem, } 4 \\
\text { Jahre mit Wieder- } \\
\text { wahl: } 40,4 \% \\
\text { jetziges Präsidialsys- } \\
\text { tem, } \\
5 \text { Jahre, einmal. } \\
\text { Amtszeit: } 31,8 \% \\
\text { semipräs. System: } \\
15,6 \% \\
\text { Kabinettsystem: } \\
11,6 \%\end{array}$ \\
\hline $\begin{array}{l}\text { Research Plus/ } \\
\text { Hankyoreh }\end{array}$ & 17.7 .2005 & $\begin{array}{l}\text { notw.: } 87,8 \% \\
\text { nicht notw.: } 9,4 \%\end{array}$ & $\begin{array}{l}\text { je eher, je besser: } \\
35,6 \% \\
\text { unter der Reg. Roh } \\
\text { Moo-hyun: } 23,6 \%\end{array}$ & $\begin{array}{l}\text { Präsidialsystem: } \\
51,6 \% \\
\text { (einmalige Amtszeit, } \\
5 \text { Jahre: } 48,6 \% \text {; } \\
4 \text { Jahre mit Wieder } \\
\text { wahl: } 35,3 \% \text { ) } \\
\text { Präs./Vizepräs: } \\
19,7 \% \\
\text { semipräs. System: } \\
14,5 \% \\
\text { Kabinettsystem: } \\
10,0 \%\end{array}$ \\
\hline
\end{tabular}

